\title{
Advances of RRAM Devices: Resistive Switching Mechanisms, Materials and Bionic Synaptic Application
}

\author{
Zongjie Shen ${ }^{1,2}$, Chun Zhao ${ }^{1,2, *(1)}$, Yanfei Qi ${ }^{1,3}$, Wangying Xu ${ }^{4}$, Yina Liu ${ }^{5}$, Ivona Z. Mitrovic ${ }^{2}$, \\ Li Yang ${ }^{6}$ and Cezhou Zhao ${ }^{1,2}$ (D) \\ 1 Department of Electrical and Electronic Engineering, Xi'an Jiaotong-Liverpool University, \\ Suzhou 215123, China; Zongjie.Shen@xjtlu.edu.cn (Z.S.); Yanfei.Qi01@xjtlu.edu.cn (Y.Q.); \\ Cezhou.Zhao@xjtlu.edu.cn (C.Z.) \\ 2 Department of Electrical Engineering and Electronics, University of Liverpool, Liverpool L69 3BX, UK; \\ Ivona@liverpool.ac.uk \\ 3 School of Electronic and Information Engineering, Xi'an Jiaotong University, Xi'an 710061, China \\ 4 College of Materials Science and Engineering, Shenzhen University, Shenzhen 518060, China; \\ wyxu@szu.edu.cn \\ 5 Department of Mathematical Sciences, Xi'an Jiaotong-Liverpool University, Suzhou 215123, China; \\ Yina.Liu@xjtlu.edu.cn \\ 6 Department of Chemistry, Xi'an Jiaotong-Liverpool University, Suzhou 215123, China; Li.Yang@xjtlu.edu.cn \\ * Correspondence: chun.zhao@xjtlu.edu.cn; Tel.: +86-(0)512-8816-1402
}

Received: 31 May 2020; Accepted: 19 July 2020; Published: 23 July 2020

\begin{abstract}
Resistive random access memory (RRAM) devices are receiving increasing extensive attention due to their enhanced properties such as fast operation speed, simple device structure, low power consumption, good scalability potential and so on, and are currently considered to be one of the next-generation alternatives to traditional memory. In this review, an overview of RRAM devices is demonstrated in terms of thin film materials investigation on electrode and function layer, switching mechanisms and artificial intelligence applications. Compared with the well-developed application of inorganic thin film materials (oxides, solid electrolyte and two-dimensional (2D) materials) in RRAM devices, organic thin film materials (biological and polymer materials) application is considered to be the candidate with significant potential. The performance of RRAM devices is closely related to the investigation of switching mechanisms in this review, including thermal-chemical mechanism (TCM), valance change mechanism (VCM) and electrochemical metallization (ECM). Finally, the bionic synaptic application of RRAM devices is under intensive consideration, its main characteristics such as potentiation/depression response, short-/long-term plasticity (STP/LTP), transition from short-term memory to long-term memory (STM to LTM) and spike-time-dependent plasticity (STDP) reveal the great potential of RRAM devices in the field of neuromorphic application.
\end{abstract}

Keywords: artificial intelligence; thin film; 2D materials; switching mechanisms; bionic synaptic application; RRAM

\section{Introduction}

In the neuromorphic system of the human brain, neuromorphic synapses are believed to be responsible for transmitting biological information. As the most typical and exquisite representative of the biological memory system, the human brain can store and process massively biological information with the adjustment of synaptic connection strength (synaptic weight) [1]. Meanwhile, they also provide complicated orthosympathetic space and energy balance [1,2]. In general, a biological synapse with the 
structure of dendrite, axon terminal and synaptic cleft is identified as a neuron linker that can permit a neuron to transmit neurotransmitters to another adjacent neuron [2]. For now, artificial synapse has attracted extensive interests and attention as the research focus in the development of artificial intelligence (AI) industry, which mainly concentrates on biomimetic synaptic functions simulated by memory functional devices in the computer industry [2]. As one of the most critical carriers for the inheritance of human civilization and the development of information technology, volatile and non-volatile memory (NVM) devices have always acted as dominating components of the development of memory devices. In the trend of miniaturization of electronic equipment, the demand for memory devices with small size, low voltage, low power consumption, and superior performance has been under extensive consideration. Currently, silicon-based flash memory, which dominates the market of data storage devices, has been difficult in meeting the needs of future development of data storage devices due to its physical and technological limitations, such as high operation voltage, high power consumption and low retention capacity [3-6]. As one of the emerging technologies of NVM, resistive random access memory (RRAM) device has been given attention as one of the next-generation memory devices. Simmons et al. reported a resistive switching (RS) characteristic in the memory device with the structure of $\mathrm{Au} / \mathrm{SiO}_{2} / \mathrm{Al}$ as early as in 1967, which provided the theoretical and experimental foundation of RRAM [7]. They demonstrated the new type of memory with the simple sandwiched structure, including top electrode (TE) layer, bottom electrode (BE) layer and an intermediate functional thin film layer (RS layer), which can be observed in Figure 1. This simple structure consisting of conductor/semiconductor or insulator/conductor makes RRAM cells be integrated into the passive crossbar array easily, with the size as small as $4 \mathrm{~F}^{2}$ (F-the minimum feature size), which can be evenly divided into $n$ parts $\left(4 \mathrm{~F}^{2} / \mathrm{n}\right)$ in the vertically stacked three-dimensional (3-D) architectures ( $\mathrm{n}$ means the stacking layer number of the crossbar array) [1-3].

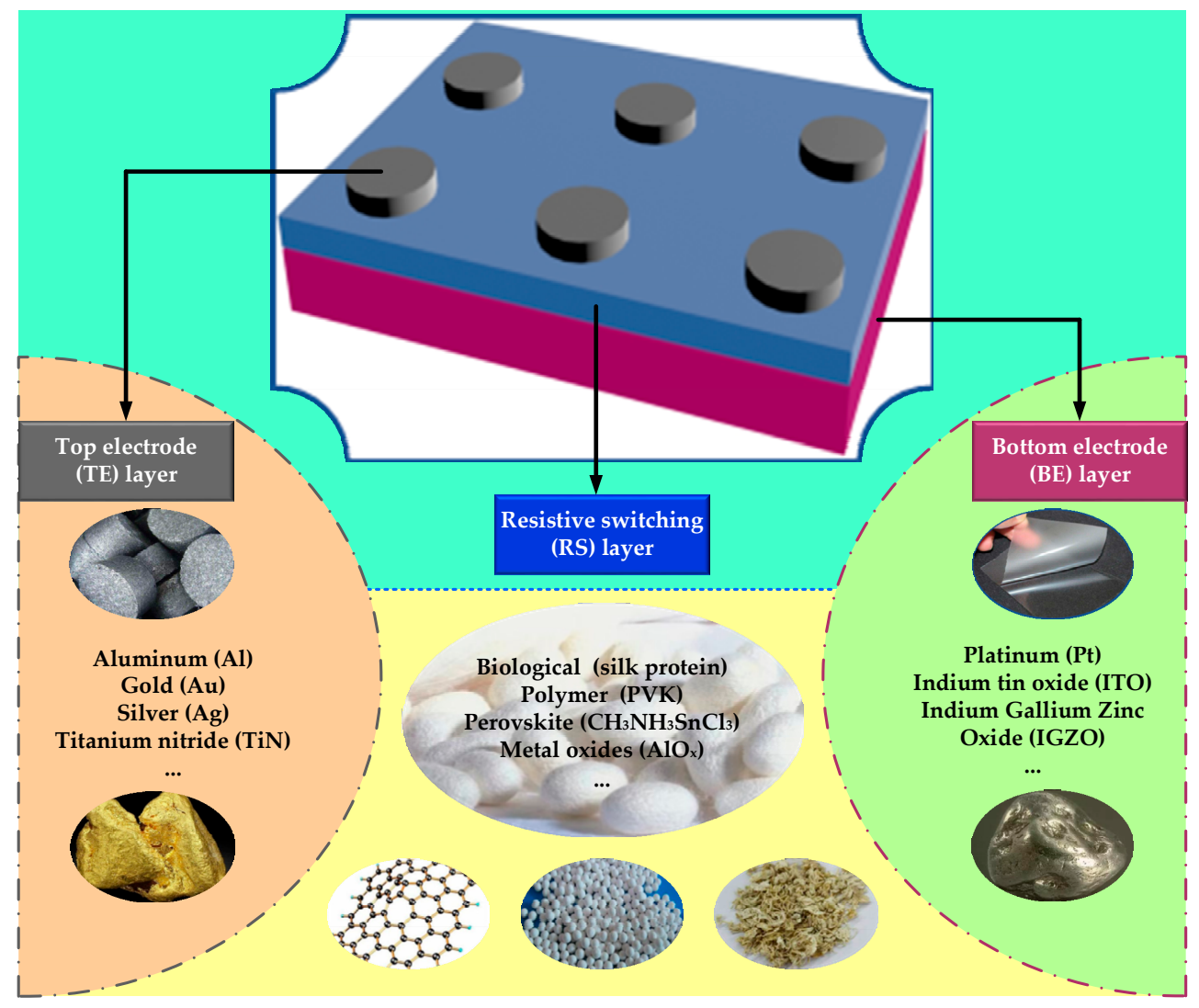

Figure 1. Illustration of sandwich structure for RRAM devices. 
The simple sandwich structure of RRAM device indicates the significance on thin film materials investigation and application. In general, performance of a RRAM device largely depends on characteristics of thin film materials for electrode and RS layers. As the main conducting medium, top and bottom electrodes are considered to enhance the electrical conductivity of RRAM devices [2]. Therefore, some thin film materials with good electrical conductivity are then chosen as candidates of the electrode, such as pure metal, semiconductor and graphene materials [1-3]. For most bipolar RRAM devices, reactive metal like titanium (Ti) [8], nickel (Ni) [9], copper (Cu) [10] and silver (Ag) [11] are always used as TE due to their high metal activity. Compared with reactive metal, where Platinum (Pt) [12] always acts as BE in order to provide activity variation of RRAM devices. In addition, semiconductor materials like heavily doped silicon and indium tin oxide (ITO) are always chosen as electrodes due to their high electrical conductivity $[13,14]$. Currently, 2D thin film materials such as graphene and graphene oxide (GO) are also attracting increasing attention as candidates of electrode because of the excellent mobility of carriers and high thermal/electrical conductivity [15-17]. Compared with electrode thin film materials, more researchers focus on thin film materials applicated on RS medium, and inorganic materials are the main investigation objectives, including oxides and solid electrolyte, which will be discussed in Chapter 3 [11,18-27].

The performances of thin-film-material-based RS layers have a decisive influence on the performance of RRAM devices, which indicates that the fabrication methods of RS layers or synthesis technologies of thin film materials cannot be neglected [1-6]. Currently, several main fabrication technologies for RS layers have received extensive recognition by researchers, such as atomic layer deposition (ALD), magnetron sputtering, chemical vapor deposition (CVD) and solution-processed deposition. For most inorganic thin film materials used on RRAM devices (such as metal oxides and solid electrolyte), ALD and sputtering are two of the most advanced technologies due to stable performance of RS layers fabricated accordingly [5-11]. In addition, our previous study indicated that some other fabrication techniques for 2D thin film materials (such as graphene, GO and hexagonal boron nitride (h-BN), $\mathrm{h}-\mathrm{BN}$ is considered as one of the most promising $2 \mathrm{D}$ materials with the function as 2D insulating template for high performance 2D electronic and photonic devices) have also been under intensive consideration, including nucleation and growth, liquid phase exfoliation and electrochemical exfoliation [21].

Apart from its typical physical structure, two basic switching states related to the conductive filament (CF) of RRAM devices, OFF and ON states, are commonly electrically characterized, which are also referred to as high-resistance-state (HRS) and low-resistance-state (LRS), respectively. With the transformation of resistance states, RRAM devices can complete the data storage process based on ' 0 ' or ' 1 '. HRS value of the device shows the low conductance state while the device demonstrates a high conductance state with LRS value. ON/OFF ratio is determined by the ratio between HRS and LRS. With the applied voltage bias, the SET operation is defined from HRS to LRS and the RESET operation is the transition from LRS to HRS. Stop voltages of SET and RESET process are defined as $\mathrm{V}_{\text {SET }}$ and $\mathrm{V}_{\text {RESET }}$. In general, two different switching types are defined as unipolar and bipolar [28-30], as illustrated in Figure 2. The unipolar switching mode is defined by the amplitude of the applied voltage bias while bipolar switching depends on the polarity of the applied voltage bias. In addition, as one of NVM devices, the endurance and retention properties of RRAM are also the presence of device reliability $[4-7,28-30]$.

Compared with conventional silicon-based memory devices like flash memory, it is noted that RRAM devices have demonstrated a series of advantages such as low operation voltage, low power consumption, high density, and enhanced compatibility with traditional complementary metal oxide semiconductor (CMOS) technology [31-33]. In addition, with the deepening of research on artificial intelligence (AI) hardware equipment, biomimetic synapse behaviors of RRAM devices have also received extensive attention, which has non-negligible influence in the investigation of electrical artificial synapse $[15,17,34-38]$. However, some other limitations and challenges of RRAM devices 
cannot be neglected, such as synthesis methods of RS materials, stability of device performance and storage mechanism of devices with different materials.

(a)

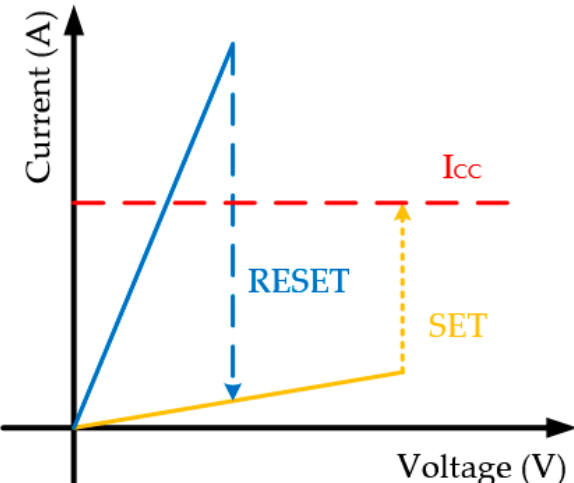

(b)
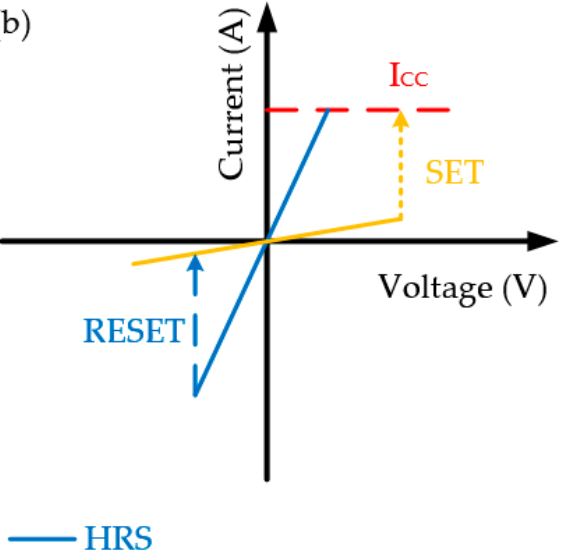

Figure 2. (a) Unipolar and (b) bipolar Modes for RRAM devices.

In this work, we provide a review of research for several main aspects, including exploration on switching mechanisms, investigation of thin film materials of RRAM devices and bionic synaptic application of RRAM devices. Section 1 provides an overview of background induction for RRAM devices. Section 2 will demonstrate a detailed discussion of different switching mechanisms. Section 3 will focus on various thin film materials applied to RRAM devices including RS medium materials and electrode materials. Section 4 will show an investigation on the neuromorphic application for RRAM devices, and Section 5 will be the conclusion of this review.

\section{Resistive Switching (RS) Mechanisms of RRAM Devices}

To present a comprehensive overview of RRAM devices, firstly, it is necessary to implement in-depth survey on different RS mechanisms of RRAM devices, which is still a controversial issue. The current investigation of RS mechanisms for sandwich structure RRAM devices is related to not only materials selection of electrode/RS medium but also utilized operation modes. For now, the most widely recognized switching mechanism is based on conductive filaments ( $\mathrm{CFs})$. However, there is no uniform and standard answer for some important issues about CFs with microscope chemical composition, physical morphology and the formation/rupture process, which have an intensive relationship to performance and working principle of RRAM devices. In this work, we will focus on the research of several working mechanisms related to anion/cation migration and thermal-chemical reaction, including thermal-chemical mechanism (TCM), valance change mechanism (VCM) and electrochemical metallization (ECM).

\subsection{Thermal-Chemical Mechanism (TCM)}

Theory about TCM can be applied to explain the formation and fracture of CFs resulted from ions (oxygen ion or metal ion) migration induced by thermal-chemical reaction (Joule heating), which is independent of the switching mode (unipolar and bipolar) for RRAM devices [39-41]. Zhang et al. explained the working principle of their Pt/Al/AlO $/ \mathrm{ITO}$ RRAM device with TCM theory [39]. As illustrated in Figure 3, oxygen ions driven by Joule heating effect drifted to TE and left oxygen vacancies in the $\mathrm{AlO}_{\mathrm{x}}$ layer; consequent $\mathrm{CFs}$ based on the accumulation of oxygen vacancies set the device to LRS. For the RESET process of unipolar device, the current steadily increased with the increasing positive voltage bias, the formed CFs finally broke when it reached the critical temperature induced by Joule heating, which made the device switch back to HRS. Similarly, for the RESET process of bipolar device, the oxygen ions drifted back to the $\mathrm{AlO}_{x}$ layer due to the melting of $\mathrm{CFs}$ and switched the device to HRS again. TCM based on Joule heating reaction is related to the formation and rupture 
of CFs. Tsuruoka et al. also proposed a research report on $\mathrm{Ag} / \mathrm{Ta}_{2} \mathrm{O}_{5} / \mathrm{Pt}$ RRAM device with TCM based on the Joule heating effect [40]. The filament based on metal Ag played a dominating role during the RS process. The formation of Ag CF made the device from HRS to LRS during the SET process. Due to the Joule-heating-based oxidation, the Ag CF ruptured by the thermal dissolution and completed the RESET operation. In general, with the SET/Forming operation, the thermal decomposition process that occurrs in the RS medium generates the ions migration in the RRAM device and the resulting formation process of CFs transforms the device from HRS to LRS. With the reversed voltage bias applied onto the electrode, the existing CFs rupture due to the thermal melting reaction, which transforms the device back to HRS and completes the RESET process accordingly.
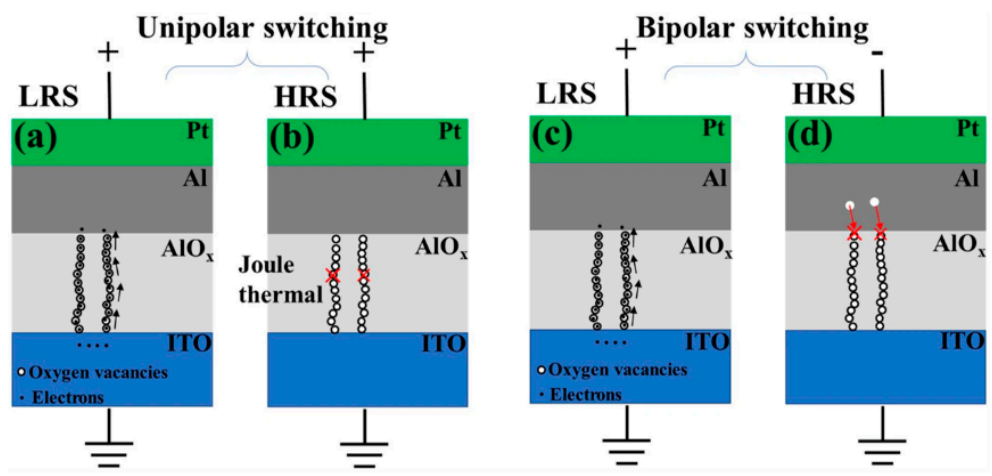

Figure 3. Switching mechanism of unipolar (a,b) and bipolar (c,d) $\mathrm{AlO}_{\mathrm{x}}$-based RRAM devices, reproduced from [39], with permission from Springer Nature, 2020.

\subsection{Valance Change Mechanism (VCM)}

Unlike the TCM mentioned above, VCM has oxygen-related defects/vacancies and their electrochemical reaction occurred in the RS medium [42-47]. In addition, it is not necessary for an RRAM device to operate with the structure that consists of an active electrode and an inert electrode, namely, the activity difference between TE and BE is not required [48]. Chen et al. researched the unipolar performance of $\mathrm{Pt} / \mathrm{SiO}_{\mathrm{x}} / \mathrm{Pt} \mathrm{RRAM}$ device with $\mathrm{VCM}$ and dangling bond (DB) [43]. As illustrated in Figure 4, with the effect of external electric field, the strength of the polar covalent $\mathrm{Si}-\mathrm{O}$ bond was weakened and finally broken. With the much higher concentration of DB near the middle of the silicon band gap, the hopping process could make the transportation of the electron through the discontinuation of DB, which was similar to the initial state (HRS) of their devices. If the DB concentration arose up to the threshold value of the percolation path, the electron transport could occur in the mini-band of DBs and the device switched into LRS, which accordingly indicated the SET process. Munjal also initiated the analysis process of $\mathrm{CoFe}_{2} \mathrm{O}_{4}$-based RRAM devices with the VCM theory [47].

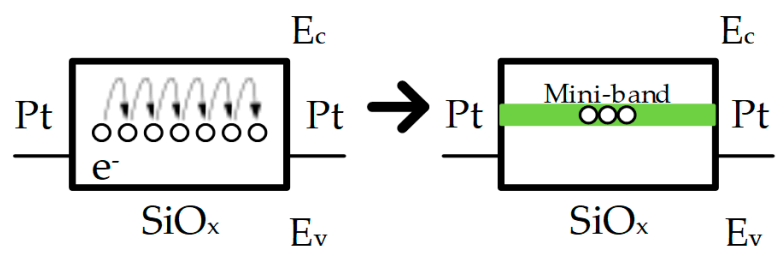

Figure 4. Schematic of discontinuous and continuous states of mini-band DBs in the middle of silicon oxide band gap.

In most cases, for VCM RRAM devices, the resistance change performance is attributed to the formation and rupture process of CF based on oxygen vacancies in the RS layer [42-47]. With the positive voltage bias applied onto the inert electrode, oxygen ions drift from where they stayed before 
with the effect of external electric field and oxygen vacancies left in the RS medium. The consequent CF path made up of leftover oxygen vacancies connects TE and BE through the functional layer, which increases the electric conductivity of the RS thin film and switches the device from HRS to LRS. Whereas, with the reversed voltage bias onto the same electrode, oxygen ions drift back to the RS layer and result in the rupture of formed CF, which makes the device switch back to HRS again. Therefore, oxygen defects/vacancies and oxygen may be the dominating aspect during the growth and destruction of CF in the functional layer.

\subsection{Electrochemical Metallization (ECM)}

Compared with TCM and VCM, ECM based on electrochemical reaction and cation migration, as the most recognized mechanism, is always used to explain the working principle of RRAM device with an active electrode, which is similar to VCM [49-55]. Generally, most active electrodes for ECM devices are active metal such as $\mathrm{Cu}$ [50-52] and Ag [49,53-55]. Tsuruoka et al. investigated $\mathrm{Cu} / \mathrm{Ta}_{2} \mathrm{O}_{5} / \mathrm{Pt} \mathrm{RRAM}$ device based on $\mathrm{Cu}$ filaments [50]. With a positive voltage bias applied onto $\mathrm{Cu} \mathrm{TE}, \mathrm{Cu}$ atoms near the interface between $\mathrm{Cu}$ layer and $\mathrm{Ta}_{2} \mathrm{O}_{5}$ layer were dissolved into $\mathrm{Cu}$ ions $\left(\mathrm{Cu}^{2+}\right)$ and electron $\left(\mathrm{e}^{-}\right)$ due to the electrochemical reaction. These $\mathrm{Cu}^{2+}$ ions drifted towards the RS layer with the effect of external electric field, which induced the $\mathrm{Cu}^{2+}$ ions supersaturation near the $\mathrm{Ta}_{2} \mathrm{O}_{5} / \mathrm{Pt}$ interface. Then continuous cathodic deposition reaction occurred between $\mathrm{Cu}^{2+}$ and $\mathrm{e}^{-}$led the formation of $\mathrm{Cu}$-based filament and switched the device into ON state.

Yu et al. also confirmed that multiple Ag filaments attributed to the multilevel RESET behavior of RRAM device with a switching layer based on nonmetal materials $\left(\mathrm{Ag} / \mathrm{SiO}_{2} / \mathrm{Pt}\right)$ [49]. With a small negative voltage bias, the $\mathrm{Ag} / \mathrm{SiO}_{2} / \mathrm{Pt}$ device exhibited gradual resistance increasement. When the voltage bias continued to increase beyond a threshold value, the resistance of device was increased to a higher state sharply, which suggested that multiple Ag filaments were effective as predicted. As demonstrated in Figure 5, in the SET process, Ag CFs with different sizes existed under a big CC after several switching cycles. During the RESET process, Ag from Ag CFs transferred into $\mathrm{Ag}^{+} \mathrm{due}^{\text {to }}$ the dissolution reaction, which resulted in a gradual resistance increase of device. When these smaller CFs were broken, the resistance changed significantly. After that, CFs with larger sizes were getting thinner until they ruptured, which further induced the multilevel performance of RESET process. Long et al. also used ECM based on Ag filament to explain the switching mechanism of $\mathrm{Ag} / \mathrm{ZrO} \mathrm{O}_{2}$ : $\mathrm{Cu} / \mathrm{Pt}$ RRAM device [53]. With the effect of external electric field induced by voltage bias applied onto $\mathrm{TE} \mathrm{Ag}$, the oxidation process occurred on $\mathrm{Ag}$ atoms and $\mathrm{Ag}$ atoms transferred into $\mathrm{Ag}$ ions $\left(\mathrm{Ag} \rightarrow \mathrm{Ag}^{+}\right.$ $+\mathrm{e}^{-}$). Then $\mathrm{Ag}^{+}$migrated gradually to $\mathrm{BE} \mathrm{Pt}$ as the electric field increased in the $\mathrm{ZrO}_{2}$ thin film and ions were reduced back to atoms $\left(\mathrm{Ag}^{+}+\mathrm{e}^{-} \rightarrow \mathrm{Ag}\right)$. Finally, the formed $\mathrm{Ag}$ filament switched the device into LRS when the voltage reached $\mathrm{V}_{\mathrm{SET}}$, which showed the related metallic transportation behavior. However, for the RESET process, when the voltage bias with reversed polarity was applied onto the active electrode, the existing Ag filament was broken within the oxide layer due to the electrochemical reaction w/o Joule heat assistance. Similarly, research reported by Tsuruoka et al. also presented the same perspective, which indicated that the RESET process related to formed metallic filaments might be related to electrochemical reaction w/o Joule heat assistance [50]. 


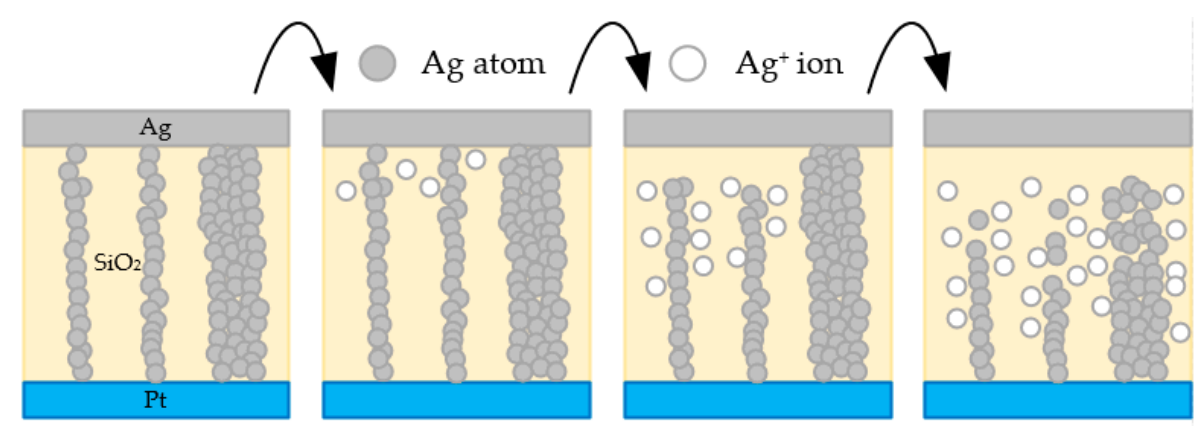

Figure 5. Illustration of multilevel RESET process of $\mathrm{Ag} / \mathrm{SiO}_{2} / \mathrm{Pt} \mathrm{RRAM}$ device.

\subsection{Modeling Analysis for Switching Mechanisms}

In order to provide convincing explanations about switching mechanisms, some researchers focused on establishing analytical models during the investigation process [41,56-61]. Ren et al. provided a specific explanation on the RS mechanism of the $\mathrm{Al} / \mathrm{CH}_{3} \mathrm{NH}_{3} \mathrm{PbI}_{3} / \mathrm{FTO}$ RRAM device, which was mainly based on a physics-based analytical model with mathematical equations [56]. In this model, the RS mechanism was generally attributed to the migration of iodine vacancy $\left(V_{I}\right)$ driven by electric field, which mainly includes operation phases such as electroforming, RESET and SET transitions. During the electroforming process, $\mathrm{V}_{\mathrm{I}}$ nucleation occurred, induced by external electric field, and then CFs were formed in the perovskite film with the voltage bias applied onto the TE. They simplified the $\mathrm{CF}$ as a cylinder with radius ( $\mathrm{r}$ ) and height $(\mathrm{h})$ and the obvious boundary existed between $\mathrm{CF}$ and outer region. In general, the region of the device where CF itself existed was the LRS region while other regions without CF indicated the HRS region. As illustrated in Figure 5, CFs themselves formed by Ag atoms (gray dots in the figure) represented LRS region while the pure wheat region without any gray dots indicated the HRS regions without CFs. With the combination of equations of Fick and Soret diffusion and related carrier drift theory, operations of RESET and SET were simulated during the switching process.

During the RESET process, $\mathrm{V}_{\mathrm{I}}$ migrated from TE to BE due to the existence of Fick diffusion, which could be regulated by equations:

$$
\begin{gathered}
J_{\text {Fick }}=D \nabla n \\
D=0.5 \alpha^{2} f \exp \left(-E_{\mathrm{a}} / k T\right)
\end{gathered}
$$

where $D$ was the diffusivity coefficient, $\nabla n$ was the concentration gradient of $\mathrm{V}_{\mathrm{I}} ; \alpha, f, E_{\mathrm{a}}, k$ and $T$ were vacancy hopping distance, escape-attempt frequency, migration activation energy, Boltzmann constant and local temperature, respectively. The gradient direction was always from BE to TE due to the function of $V_{I}$ reservoir for $B E$, thus the RESET process was expected to be confined by the Fick diffusion. Apart from Fick diffusion, another vital factor, the voltage-field activated $V_{I}$ drift could not be neglected, which could be expressed by equations:

$$
\begin{gathered}
J_{\text {drift }}=-v n \\
v=a f \exp \left(-E_{\mathrm{a}} / k T\right) \sinh (q a E / 2 k T)
\end{gathered}
$$

where $v$ was the drift velocity, $E$ was the electric field inside the $C F$, and $q$ was elementary charge. With the combination of $J_{\text {Fick }}$ and $J_{\text {drift }}$, the time evolution of CF size could be expressed by equation:

$$
\frac{d g}{d t}=D \alpha_{1} e^{-\frac{\beta_{1}}{0.5(L-g)}}-v
$$

where $L$ is the thickness of perovskite film, and $g$ is the gap length. In addition, the Joule heating flow that occurred in the lateral direction of CF was also noted, which had a closed relationship with the 
length and diameter of $\mathrm{CF}$. For the SET process, $\mathrm{V}_{\mathrm{I}}$ migration occurred from BE to TE with the effect of reversed applied voltage bias resulting in the refill of the gap region. They explained the phenomenon with a set kinetics model based on Fick and Soret diffusion. Similar to Fick diffusion in the RESET process, the formation and growth of CFs were also suppressed by Soret diffusion in SET process, which was associated with the migration tendency of $\mathrm{V}_{\mathrm{I}}$ towards the region with higher temperature.

According to their simulation results, the electric field inside CF decreased gradually when the depletion process occurred and then induced the slowing down of gap formation, which was used to explain why the growth of gap length mainly occurred in the positive part of voltage sweep. Apart from mathematical modeling, another main technique is modeling with software to justify the switching mechanisms. Software like COMSOL was also used to establish physics-based analytical models for further analysis $[41,60]$. Zhou et al. proposed a COMSOL-based model to research the switching mechanism of the Ti/ $/ \mathrm{HfO}_{2} / \mathrm{TiN}$ RRAM device [60]. They chose the 1st, 10th and 20th cycles as fitting analysis objectives, which all performed with Schottky emission. Based on the Schottky fitting formula, the gradual larger Schottky distance and the barrier had a relationship with a larger intercept and the slope of $\operatorname{Ln}(\mathrm{I})-(\mathrm{V})^{1 / 2}$. According to the simulation results, the strongest electric field that existed in the tip of CF contacted the electrode in the initial SET process. After that, with more SET and RESET operations, oxygen ions accumulation occurred in the interface between $\mathrm{TiN}$ and $\mathrm{HfO}_{2}$ layers, which made SET and RESET more difficult to achieve. Sun et al. also reported a similar mechanism on $\mathrm{Cu} / \mathrm{ZrO}_{2} / \mathrm{Pt}$ RRAM device [41].

\section{Thin Film Materials of RRAM Devices}

Apart from different RS switching mechanisms, most researches also put effort into the study of various thin film materials applied in RRAM devices. Before we present an investigation of materials application, it is necessary to provide brief induction of figures of merit (FoMs), which are mainly used to assess the performance of RRAM devices, including operation speed, power consumption, reliability, scalability, and cost [62]. Operation speed is defined by random-access time and effective time of write \& erase ( $w$ \& e) speed of a single RRAM cell; power consumption is affected by static and dynamic power consumption; reliability consists of endurance; retention properties are used to determine whether an RRAM device is reliable; scalability of an RRAM device determines whether RRAM devices can be developed in line with current trends of increasing device density; and the cost directly has an impact on marketization or mass-production of the proposed devices [62]. Apart from the main FoMs presented above, some other specific characteristics of FoMs such as SET/RESET voltage, ON/OFF switching ratio, distribution of operation voltage, and resistance cannot be neglected accordingly.

Thin film materials application of RRAM devices can be divided into two directions: materials for RS medium and materials for electrode (TE/BE). Materials of RS medium play a decisive role in the switching process, which has a direct and significant influence on the performance of RRAM devices such as ON/OFF ratio and device stability. On the other hand, electrode materials of RRAM devices more affect switching modes of RRAM devices, which should also be under further investigation $[13,14,18,48,63,64]$.

\subsection{Thin Film Materials of RS Medium}

A lot of thin film materials have been researched as RS mediums of RRAM devices due to their exhibition of RS characteristics with the effect of external electric field. In general, organic materials and inorganic materials are two main categories of RS medium, as illustrated in Figure 6. Research of RS medium based on organic materials mainly focuses on biological materials (silk protein/fibroin, nanocellulose and albumen) [65-67], polymer materials (PVK (polyvinyl carbazole), PVA (polyvinyl alcohol), PDA (Polydiacetylene), PTH (polythiophene)) [68-71], and other materials. Chen et al. presented an RRAM device fabricated with spin-coated chicken egg albumen layer [67]. With the low SET/RESET voltage $\sim 3 \mathrm{~V}$, the reliable switching endurance was observed over 500 cycles with $\sim 10^{3}$ ON/OFF ratio. Similarly, Wang et al. reported an RRAM device with a structure of 
$\mathrm{Au} / \mathrm{Mg} /$ fibroin/Mg and the fibroin-based RS layer was fabricated with drop-casting method [65]. Their device exhibited excellent performance with operation voltage lower than $\sim 2 \mathrm{~V}$, ON/OFF ratio higher than $\sim 10^{3}$ and retention time longer than $\sim 10^{4} \mathrm{~s}$. In addition, they evaluated the transient behaviors of RRAM devices with the immersion process in DI water, which indicated the great potential of silk fibroin applied to transient and biocompatible electronics. Although the increasing number of research studies has demonstrated the feasibility of RRAM devices with organic materials, high power consumption under high operation voltage and dispersed voltage/resistance distribution induced by disappointing stability and reliability of device fabricated with organic materials indicated that it is necessary to further investigate perfect application of organic materials in the industry of memristive devices. In the future, most of these organic materials will be considered as probable candidates of application in flexible and wearable memristive devices with health diagnosis monitoring as the main function.

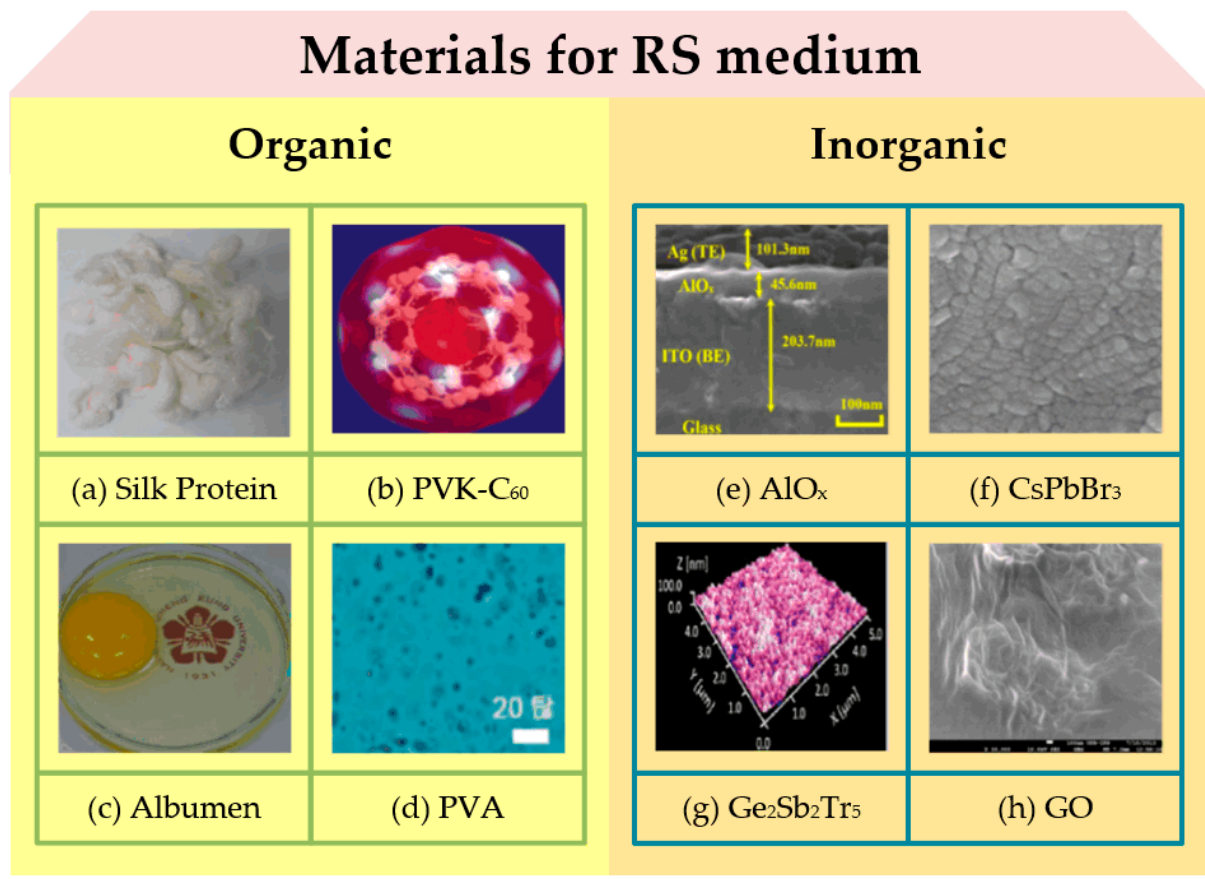

Figure 6. Various thin film materials of RS medium for RRAM devices. (a) Silk protein, reproduced from [65], with permission from John Wiley and Sons, 2016. (b) PVK-C 60 , reproduced from [72], with permission from American Chemical Society, 2007. (c) Albumen, reproduced from [67], with permission from Springer Nature, 2015. (d) PVA, reproduced from [71], with permission from John Wiley and Sons, 2011. (e) $\mathrm{AlO}_{\mathrm{x}}$, reproduced from [19], with permission from Elsevier, 2020. (f) $\mathrm{CsPbBr}_{3}$, reproduced from [73], with permission from John Wiley and Sons, 2019. (g) $\mathrm{Ge}_{2} \mathrm{Sb}_{2} \mathrm{Tr}_{5}$, reproduced from [38], with permission from John Wiley and Sons, 2019. (h) GO, reproduced from [74], with permission from AIP Publishing, 2013.

Compared with RRAM devices fabricated with organic materials, better electrical performance of RRAM devices based on inorganic materials can be observed with more stable switching behavior, lower energy consumption and longer retention time. Inorganic materials are also being given extensive attention due to their simple manufacturing process and superior properties. In this review, we will present RRAM-related inorganic materials with oxides, solid electrolyte and two-dimensional (2D) materials. Table 1 demonstrated a performance comparison among RRAM devices with different oxide layers, including binary and complex oxide materials. As one of the most typical representatives of oxides, binary oxides have been explored for over half a century due to their simple composition, low manufacture cost, compatibility with traditional CMOS (complementary metal-oxide-semiconductor transistor) technology, and ease of fabrication and control. Among binary 
oxides, binary metal oxides such as $\mathrm{Al}_{2} \mathrm{O}_{3}[9,20,75]$, $\mathrm{NiO}[8,12,76], \mathrm{TiO}_{2}[21,77,78], \mathrm{HfO}_{2}[60,79,80]$, $\mathrm{ZnO}$ [81-83], and $\mathrm{ZrO}_{2}[22,84,85]$ are always playing main roles in materials application of RS medium.

Table 1. Performance comparison among RRAM devices with various binary and complex oxides (metal and nonmetal materials).

\begin{tabular}{|c|c|c|c|c|c|c|c|c|c|}
\hline Structure & $\begin{array}{c}\text { Switching } \\
\text { Mode }\end{array}$ & $\begin{array}{c}\text { Thickness } \\
\text { (nm) }\end{array}$ & $\begin{array}{l}\text { V Forming }_{\text {Fo }} \\
\text { (V) }\end{array}$ & $\begin{array}{l}V_{\text {SET }} \\
\text { (V) }\end{array}$ & $\begin{array}{l}\mathrm{V}_{\text {RESET }} \\
(\mathrm{V})\end{array}$ & $\begin{array}{c}\text { ON/OFF } \\
\text { Ratio }\end{array}$ & $\begin{array}{c}\text { Endurance } \\
\text { (cycle) }\end{array}$ & $\begin{array}{l}\text { Retention } \\
\text { (s) }\end{array}$ & Ref. \\
\hline $\mathrm{Ni} / \mathrm{AlO}_{\mathrm{x}} / \mathrm{Pt}$ & bipolar & $\sim 40$ & Free & $\sim 1.0$ & $\sim-1.0$ & $\sim 10^{3}$ & $>150$ & $>10^{4}$ & [9] \\
\hline $\begin{array}{l}\mathrm{TaN} / \mathrm{HfO}_{2} / \\
\mathrm{Al}_{2} \mathrm{O}_{3} / \mathrm{ITO}\end{array}$ & bipolar & $\sim 6$ & $\sim 4.5$ & $\sim 1.5$ & $\sim-1.0$ & $\sim 10^{2}$ & $>100$ & $>2 \times 10^{3}$ & [20] \\
\hline $\mathrm{Ti} / \mathrm{IL}-\mathrm{NiO} / \mathrm{Pt}$ & bipolar & $\sim 50$ & Free & $\sim 0.5$ & $\sim-1.5$ & $\sim 10^{3}$ & $>1300$ & $>10^{4}$ & [8] \\
\hline $\mathrm{FeNi} / \mathrm{Al}_{2} \mathrm{O}_{3} / \mathrm{NiO} / \mathrm{Pt}$ & bipolar & $\sim 180$ & $\sim 4.07$ & $\sim 6.0$ & $\sim-5.0$ & $\sim 10^{3}$ & $>100$ & $>10^{4}$ & [12] \\
\hline $\begin{array}{l}\mathrm{Au} / \mathrm{TiO}_{\mathrm{x}} / \\
\mathrm{TiO}_{\mathrm{y}} / \mathrm{Au}\end{array}$ & bipolar & $\sim 50$ & $\sim 5.62$ & $\sim 1.0$ & $\sim-2.0$ & $\sim 10^{2}$ & N. A. & N. A. & [78] \\
\hline $\begin{array}{l}\mathrm{Ni} / \mathrm{SiGeO}_{\mathrm{x}} / \\
\mathrm{TiO}_{\mathrm{y}} / \mathrm{TiN}\end{array}$ & bipolar & $\sim 25$ & Free & $\sim 3.0$ & $\sim-2.5$ & $\sim 10^{3}$ & $>10^{4}$ & $>10^{5}$ & [21] \\
\hline $\mathrm{Ti} / \mathrm{HfO}_{2} / \mathrm{TiN}$ & bipolar & $\sim 15$ & $\sim 6.5$ & $\sim 1.0$ & $\sim-0.8$ & $\sim 10$ & N. A. & N. A. & [60] \\
\hline $\mathrm{Pt} / \mathrm{Hf} / \mathrm{HfO}_{2} / \mathrm{TiN}$ & bipolar & $\sim 20$ & Free & $\sim 0.8$ & $\sim-1.5$ & $\sim 10^{2}$ & N. A. & $>10^{6}$ & [80] \\
\hline $\mathrm{Pt} / \mathrm{Ta} / \mathrm{HfO}_{2} / \mathrm{TiN}$ & bipolar & $\sim 20$ & Free & $\sim 0.8$ & $\sim-1.8$ & $\sim 10^{2}$ & N. A. & $>10^{4}$ & [80] \\
\hline $\mathrm{Pt} / \mathrm{Al}: \mathrm{HfO}_{2} / \mathrm{TiN}$ & bipolar & $\sim 9$ & $\sim 2.3$ & $\sim 2.0$ & $\sim-2.0$ & $\sim 10^{4}$ & $>100$ & $>10^{4}$ & [32] \\
\hline $\begin{array}{l}\mathrm{TiN} / \mathrm{ZnO} / \\
\mathrm{TiN}\end{array}$ & bipolar & $\sim 9$ & $\sim 4.2$ & $\sim 1.0$ & $\sim-1.0$ & $\sim 10$ & 240 & N. A. & [82] \\
\hline $\begin{array}{c}\mathrm{TiN} / \mathrm{Al}_{2} \mathrm{O}_{3} / \\
\mathrm{ZnO} / \mathrm{Al}_{2} \mathrm{O}_{3} / \mathrm{TiN} \\
\end{array}$ & bipolar & $\sim 15$ & $\sim 5.0$ & $\sim 1.0$ & $\sim-1.0$ & $\sim 10^{2}$ & $>10^{4}$ & $>10^{4}$ & [82] \\
\hline $\mathrm{ITO} / \mathrm{ZrO}_{2} / \mathrm{Ag}$ & bipolar & $\sim 50$ & N. A. & $\sim 5.0$ & $\sim-15.0$ & $\sim 10^{5}$ & $>100$ & $>10^{4}$ & [22] \\
\hline $\begin{array}{c}\mathrm{Pt} / \mathrm{N}: \mathrm{ZrO}_{2} / \\
\mathrm{TiN}\end{array}$ & bipolar & $\sim 25$ & $\sim 3.6$ & $\sim 0.5$ & $\sim-1.0$ & $\sim 10^{2}$ & N. A. & N. A. & [85] \\
\hline $\mathrm{Ag} / \mathrm{SiO}_{2} / \mathrm{Pt}$ & bipolar & $\sim 80$ & N. A. & $\sim 0.5$ & $\sim-2.0$ & $\sim 10^{6}$ & $>40$ & $>2 \times 10^{3}$ & [49] \\
\hline $\mathrm{ITO} / \mathrm{LaAlO}_{3} / \mathrm{ITO}$ & bipolar & $\sim 30$ & $\sim 3.2$ & $\sim 3.0$ & $\sim-3.0$ & $\sim 10^{2}$ & $>100$ & N. A. & [86] \\
\hline $\mathrm{Cu} / \mathrm{Cu}: \mathrm{LaAlO}_{3} / \mathrm{Pt}$ & bipolar & $\sim 10$ & $\sim 7.0$ & $\sim 2.0$ & $\sim-2.0$ & $\sim 10^{3}$ & $>110$ & $>10^{4}$ & [10] \\
\hline $\mathrm{GNR} / \mathrm{SrTiO}_{3} / \mathrm{GNR}$ & bipolar & $\sim 50$ & N. A. & $\sim 2.0$ & $\sim-3.0$ & $\sim 10$ & $>200$ & $>10^{4}$ & [87] \\
\hline $\mathrm{Pt} / \mathrm{GO} / \mathrm{PCMO} / \mathrm{Pt}$ & bipolar & $\sim 25$ & Free & $\sim 1.0$ & $\sim-1.0$ & $\sim 10^{2}$ & $>150$ & $>10^{4}$ & [23] \\
\hline $\mathrm{Pt} / \mathrm{BiFeO}_{3} / \mathrm{Pt}$ & unipolar & $\sim 200$ & N. A. & $\sim 5.0$ & $\sim-15.0$ & N. A. & N. A. & N. A. & [88] \\
\hline $\mathrm{Ag} / \mathrm{ZnO} / \mathrm{BiFeO}_{3} / \mathrm{ZnO} / \mathrm{Ag}$ & bipolar & $\sim 270$ & Free & $\sim 2.0$ & $\sim-2.0$ & $\sim 10$ & N. A. & N. A. & [11] \\
\hline $\begin{array}{c}\mathrm{Ag} / \mathrm{Ag}_{2} \mathrm{Se} / \\
\mathrm{MnO} / \mathrm{Au}\end{array}$ & bipolar & $\sim 40$ & Free & $\sim 0.8$ & $\sim-0.6$ & $\sim 10^{2}$ & $>800$ & $>10^{4}$ & [24] \\
\hline $\mathrm{TiN} / \mathrm{SLG} / \mathrm{HfO}_{2} / \mathrm{Pt}$ & bipolar & $\sim 35$ & $\sim 5.0$ & $\sim 2.0$ & $\sim-3.0$ & $\sim 10^{2}$ & $>120$ & $>10^{6}$ & [89] \\
\hline Ti/MoS 2 -rGO/ITO & bipolar & $\sim 60$ & Free & $\sim 0.5$ & $\sim-0.4$ & $\sim 10$ & $>200$ & $>10^{4}$ & [26] \\
\hline $\mathrm{Au} / \mathrm{CsPbBr}_{3} / \mathrm{ITO}$ & bipolar & N, A. & Free & $\sim 1.0$ & $\sim-1.0$ & $\sim 10^{4}$ & N. A. & $>1200$ & [90] \\
\hline
\end{tabular}

The first report of RS performance in binary metal oxides was proposed by Hickmott in 1962 [91], which demonstrated the RS characteristics of $\mathrm{Al} / \mathrm{Al}_{2} \mathrm{O}_{3} / \mathrm{Al}$ device under the effect of an electric field. With the development of fabrication methods for electronic devices with thin films, binary metal oxides thin films fabricated by sputtering, ALD (atomic layer deposition) and solution-processed methods have received more interest due to their superior performance. Also, it is indicated that some researchers not only focus on RS layer fabricated with conventional pure binary metal oxides, but also explore effective ways of optimization treatment on metal-oxides-based RS layers, such as stack layers, ionic liquid (IL) process and nanoparticle (NP) doping process, and other similar treatment methods [8,20,32].

Mahata et al. reported an RRAM device with ALD-based $\mathrm{HfO}_{2} / \mathrm{Al}_{2} \mathrm{O}_{3}$ stack layers, which exhibited excellent performance with operation voltage lower than $\sim 2 \mathrm{~V}$ and the ON/OFF ratio around $\sim 10^{3}$. The TaN/ $/ \mathrm{HfO}_{2} / \mathrm{Al}_{2} \mathrm{O}_{3} / \mathrm{ITO}$ RRAM device also presented neuromorphic synaptic behaviors with multi-level conductance properties by tuning the stop voltage in a DC sweep and the amplitude in pulse responses [20]. Shen et al. presented various RRAM devices with solution-processed $A_{10}$ layers annealed at different temperatures [9]. As illustrated in Figure 7, their research results on $\mathrm{Ni} /$ solution-processed $\mathrm{AlO}_{\mathrm{x}} / \mathrm{Pt}$ RRAM devices, indicating that the best performance can be found in the device with a solution-processed $\mathrm{AlO}_{\mathrm{x}}$ layer annealed at $250{ }^{\circ} \mathrm{C}$, which exhibited operation voltage around $\sim 1 \mathrm{~V}, \mathrm{ON} / \mathrm{OFF}$ ratio higher than $\sim 10^{3}$, endurance cycles over 100, and retention time longer 
than $10^{4} \mathrm{~s}$. Samanta et al. reported the threshold switching performance of their cross-point selector with $\mathrm{Al}_{2} \mathrm{O}_{3}$ and $\mathrm{SiO}_{\mathrm{x}}$ as bilayer dielectric [92]. Compared with the cross-point selector with only sputtering-deposited $\mathrm{SiO}_{x}$ layer, the ALD-deposited $\mathrm{Al}_{2} \mathrm{O}_{3}$ layer in the bilayer platform controlled the dissolution gap of Ag filament and improved the uniformity of the device. The selectivity was larger than $5 \times 10^{7}$ and the rectifying ratio (RR) was over $\sim 10^{7}$. Other researchers like Banerjee, Knorr and Sleiman et al. also demonstrated their investigation on RS behaviors of $\mathrm{AlO}_{\mathrm{x}}$-based RRAM devices [93-97].
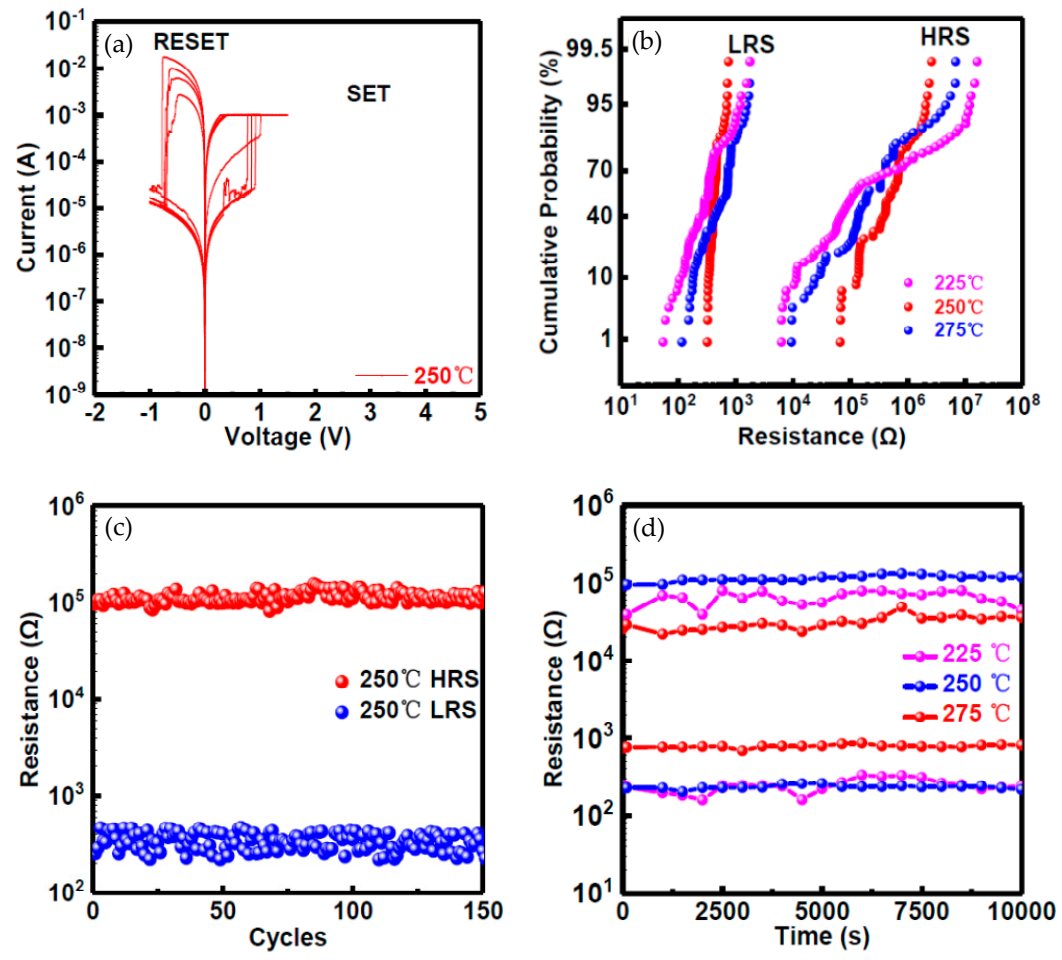

Figure 7. RS performance, including (a) bipolar I-V characteristic, (b) resistance distribution, (c) endurance and (d) retention performance, of $\mathrm{Ni} /$ solution-processed $\mathrm{AlO}_{\mathrm{x}} / \mathrm{Pt} \mathrm{RRAM}$ devices annealed at different temperatures, reproduced from [9], with permission from MDPI (Basel, Switzerland), 2019.

Kang et al. proposed a Ti/IL-NiO/Pt RRAM device with IL-treated NiO layer, which made the RRAM device operated under only $\sim 0.5 \mathrm{~V}$ voltage bias with ON/OFF ratio higher than $\sim 10^{3}$ [8]. The IL treatment on $\mathrm{NiO}$ thin film created $\mathrm{Ni}^{0}$-regions near the $\mathrm{NiO} / \mathrm{Pt}$ interface and was helpful to the formation of oxygen vacancy filament, which improved device performance. The self-assembled memristive element based on $\mathrm{NiO}$ nanocrystal arrays was proposed by Kurnia et al. [98]. NiO nanocrystals were synthesized onto a $\mathrm{SrRuO}_{3}$ substrate with PLD (pulsed laser deposition) using the phase separation method. Their devices exhibited memristive switching behavior with nonlinear bipolar characteristics under the $\sim 5 \mathrm{~V}$ operation voltage, which was investigated via scanning probe microscopy, based on first-order reversal curve current-voltage spectroscopy. In addition, it was indicated that low electrical dissipation at the edge of the nanocrystals represented that less energy was consumed as heat, which enhanced the utilization efficiency during the nucleation process of CFs and reduced the energy consumption. Besides, further exploration on RS performance of $\mathrm{NiO}$ dielectric was also carried out by Yoshida, Russo, Cagli, and Ielmini et al. [99-103].

Kim et al. reported an RRAM device with a structure of $\mathrm{Au} / \mathrm{TiO}_{\mathrm{x}} / \mathrm{TiO}_{\mathrm{y}} / \mathrm{Au}$, and the $\mathrm{TiO}_{\mathrm{x}} / \mathrm{TiO}_{\mathrm{y}}$ layers were fabricated by the sputtering method [78]. During the experimental process, their modified operations on the gas environment made $\mathrm{TiO}_{x}$ and $\mathrm{TiO}_{y}$ layers deposited under a gas mixture of Ar and $\mathrm{O}_{2}$ with flow ratios of 20:5 and 20:1, respectively. The $\mathrm{Au} / \mathrm{TiO}_{\mathrm{x}} / \mathrm{TiO}_{\mathrm{y}} / \mathrm{Au} \mathrm{RRAM}$ device operated under $\sim 1.5 \mathrm{~V}$ with $\sim 10^{2} \mathrm{ON} / \mathrm{OFF}$ ratio, which also exhibited artificial synaptic characteristics 
such as long-term potentiation (LTP), long-term depression (LTD), and spike-timing-dependent plasticity (STDP). Mullani et al. reported the enhanced RS behavior of their devices based on hydrothermal-fabricated carbon nanotube/ $\mathrm{TiO}_{2}$ nanorods composite film through increasing oxygen vacancy reservoir [4]. The effect of concentration of $\mathrm{TiO}_{2}-\mathrm{fMWCNT}$ (functionalized multiwalled carbon nanotube) nanocomposites was confirmed, which improved the RS performance of the device with forming-free and low operational voltage when the concentration of fMWCNT was $0.03 \mathrm{wt} \%$. Sakellaropoulos et al. demonstrated a comparison among different devices with three kinds of dielectric structures such as $\mathrm{HfO}_{x}, \mathrm{TaO}_{y} / \mathrm{HfO}_{x}$ and $\mathrm{HfO}_{x} / \mathrm{TaO}_{y} / \mathrm{HfO}_{x}$, which were corresponding to single-layer (SL), bilayer (BL) and triple-layer (TL) [104]. The forming-free sample TL exhibited enhanced RS behavior with ON/OFF ratio larger than $\sim 10^{2}$, cycling variability smaller than 0.6 and number of endurance cycles over $10^{6}$ under only $\sim \mathrm{nW}$-level operation power in pulsing mode. Compared with sample SL, the RS performance of sample TL was confined due to the higher oxygen content and deeper oxygen vacancy levels of the $\mathrm{TaO}_{\mathrm{y}}$ layer, which demonstrated analog switching characteristics and revealed great potential in artificial synaptic application. Besides, other researchers such as Nauenheim, Hermes, Salaoru and Otsuka et al. also provided a related investigation on $\mathrm{TaO}_{\mathrm{x}}-$ and $\mathrm{TiO}_{\mathrm{x}}-\mathrm{based}$ RRAM devices in terms of electrical performance and physical characterization [105-109].

Wang et al. proposed an interface engineering method on ALD-based $\mathrm{HfO}_{2}$ thin film with $\mathrm{O}_{3}$, which improved the performance of $\mathrm{Pt} / \mathrm{HfO}_{2} / \mathrm{TiN}$ RRAM device [79]. It is reported that the best stability could be observed in the $\mathrm{HfO}_{2}$ RRAM device with 20 pulses of $\mathrm{O}_{3}$ treatment. The TiON layer was observed at the interface between $\mathrm{HfO}_{2}$ and TiN layers. With the voltage bias onto electrode, more abundant detects survived at the TiON layer due to the longer oxidation process, which had a positive influence on the migration efficiency of oxygen vacancy and formation of CFs. However, it also resulted in the obvious augmentation of the conductivity of the $\mathrm{HfO}_{2}$ layer. In addition, their research on neuromorphic simulation based on potentiation, depression and STDP emulated the presynaptic and postsynaptic membranes of a biological synapse through applying the same pulses on both $\mathrm{Pt}$ and TiN electrodes, which revealed that oxide/metal interface engineering could have significant impact on RS characteristics of RRAM devices. Sharath et al. reported their RRAM devices based on RMBE (reactive molecular beam epitaxy)-deposited $\mathrm{HfO}_{2-x}$ layer with the operation voltage lower than $1 \mathrm{~V}$ and ON/OFF ratio higher than $10^{2}$ [110]. With the Hard X-ray photoelectron spectroscopy, the presence of sub-stoichiometric hafnium oxide and defect states near the Fermi level were confirmed. Bipolar RS performance was also observed on forming-free RRAM devices with oxygen-deficient $\mathrm{HfO}_{2-x}$ thin films. Besides, other related research on RS behaviors of $\mathrm{HfO}_{\mathbf{x}}$ dielectric thin films were also reported by Clima, Lanza and Stefano et al. [111-114].

$\mathrm{Ha}$ et al. demonstrated an $\mathrm{Ag} / \mathrm{ZrO}_{2} / \mathrm{ITO}$ RRAM device with sol-gel-processed $\mathrm{ZrO}_{2}$ thin film, which exhibited excellent performance with operation voltage around $\sim 2 \mathrm{~V}$ and ON/OFF ratio higher than $10^{5}$ [22]. They investigated the effect of different annealing gas environments (air, vacuum and $\mathrm{N}_{2}$ ) on the performance of RRAM devices. Sol-gel-processed $\mathrm{ZrO}_{2}$ thin film annealed at vacuum showed enhanced performance with larger crystallinity and grain size, denser film, and a relatively small quantity of oxygen vacancies, which resulted in a decrease in the leakage current and an increase in the resistance ratio of HRS/ LRS and successfully improved non-volatile memory properties, such as endurance and retention characteristics. Abbas et al. proposed their investigation regarding $\mathrm{Ti} / \mathrm{ZrO}_{\mathrm{x}} / \mathrm{Pt}$ RRAM device with RTA (post-rapid thermal annealing) processing on $\mathrm{ZrO}_{\mathrm{x}}$ dielectric layer [115]. Compared with samples based on an as-deposited $\mathrm{ZrO}_{\mathrm{x}}$ layer without annealing process, RTA sample demonstrated improved RS performance such as lower operation voltage, higher RS ratio, longer retention time, and increasing number of endurance cycles. Particularly, with the annealing temperature of $700{ }^{\circ} \mathrm{C}$ during the RTA process, $\mathrm{Ti} / \mathrm{ZrO}_{\mathrm{x}} / \mathrm{Pt} \mathrm{RRAM}$ device could operate with a voltage lower than $1 \mathrm{~V}$ and ON/OFF ratio higher than $10^{3}$. Apart from Ha and Abbas, Verbakel, Awais, Kärkkänen and Ismail et al. also exhibited their investigation on RRAM devices with $\mathrm{ZrO}_{\mathrm{x}}$ dielectric layers and most of them fabricated $\mathrm{ZrO}_{x}$ thin films with ALD and sputtering methods [116-120]. 
Similar to binary metal oxides, binary nonmental oxides like $\mathrm{SiO}_{2}$ were also under investigation [49]. $\mathrm{Yu}$ et al. reported the multi-level RS performance of $\mathrm{Ag} / \mathrm{SiO}_{2} / \mathrm{Pt} \mathrm{RRAM}$ devices with operation voltage smaller than $1.5 \mathrm{~V}$ and switching ratio higher than $10^{2}$, which was affected by the formation of multiple Ag filaments [49]. Apart from binary oxides, complex oxides with higher dielectric constants, such as $\mathrm{LaAlO}_{3}[10,86], \mathrm{SrTiO}_{3}[87,121], \mathrm{Pr}_{0.7} \mathrm{Ca}_{0.3} \mathrm{MnO}_{3}[23,122]$ and $\mathrm{BiFeO}_{3}[11,88]$ are also explored to improve the switching performance of RRAM devices. Bailey et al. exhibited a stack-layered RRAM device with a structure of $\mathrm{W} / \mathrm{SrTiO}_{3}(\mathrm{STO}) / \mathrm{TiN}$, which systematically investigated the diffusion phenomenon of ionic defects in oxides associated with various configuration states of STO layer. Consistent decay was found during the test of device retention, and then the function relationship between decay rate and conditioning voltage was confirmed [121]. The metal/ $\operatorname{Pr}_{0.7} \mathrm{Ca}_{0.3} \mathrm{MnO}_{3}(\mathrm{PCMO}) / \mathrm{metal} \mathrm{RRAM}$ devices with a high-throughput electrode were studied by Tsubouchi et al. based on various metal electrodes including Mg, Ag, Al, Ti, Au, Ni, and Pt [122]. Typical RS behaviors were only observed on devices with $\mathrm{Al}$ electrode in the measurement process of $\mathrm{I}-\mathrm{V}$ and pulsed-field resistance. In addition, the switching performance was always found near the Al/PCMO interface with the test results of the four-probe test.

Except binary and complex oxides, solid electrolyte and 2D materials are also popular in the research on RRAM-related inorganic materials. As early as in 1976, $\mathrm{As}_{2} \mathrm{~S}_{3}$ solid electrolyte material has been studied as a functional layer in the RRAM device by Hirose et al. [123]. With the photodoping operation of $\mathrm{Ag}$ ions into the RS layer, the RRAM device with the $\mathrm{Ag}-\mathrm{As}_{2} \mathrm{~S}_{3}$ layer operated under low voltage, and CF based on the Ag element was observed by an optical microscope. After that, some solid electrolyte materials have been presented including $\mathrm{Ag}_{2} \mathrm{Se}$ [24], $\mathrm{Ge}_{2} \mathrm{Sb}_{2} \mathrm{Te}_{5}$ [25], GeTe [124], etc. At present, a series of 2D materials such as graphene $[89,125,126]$, molybdenum disulphide $\left(\mathrm{MoS}_{2}\right)[26,127,128]$ and perovskite materials $\left(\mathrm{CH}_{3} \mathrm{NH}_{3} \mathrm{SnCl}_{3}\right.$ and $\left.\mathrm{CsPbBr} 3\right)[73,90]$ also inspired researchers' interest due to their small size, ultra-thin thickness and excellent physical properties, which have resulted in superior performance of RRAM devices. Chen et al. proposed an electrode/oxide interface engineering technique by inserting single-layer graphene (SLG) into the TiN/HfO $/ 2 / \mathrm{Pt} \mathrm{RRAM}$ device [89], which enabled the RESET current to be reduced by 22 times and the programming energy consumption reduced by 47 times. Raman mapping and single-point measurement on the SLG layer noted that signals of D-band and G-band changed during the electrical cycling, which indicated that oxygen ions might drift from metal oxide layer to SLG layer. Wu et al. exhibited a flexible RRAM device based on $\mathrm{MoS}_{2}-\mathrm{rGO}$ (reduced graphene oxide) hybrid layer synthesized by hydrothermal reaction between $\left(\mathrm{NH}_{4}\right)_{6} \mathrm{Mo}_{7} \mathrm{O}_{24} \cdot 4 \mathrm{H}_{2} \mathrm{O}$ and $\left(\mathrm{NH}_{2}\right)_{2} \mathrm{CS}$ in $\mathrm{GO}$ (graphene oxide) dispersion with the distilled water as dispersant [26]. As illustrated in Figure 8, their device showed not only a low SET/RESET voltage $(\sim 0.4 \mathrm{~V})$ but also multi-level states through compliance current (CC) control. Excellent electrical performance could also be observed with endurance cycles over 200, retention time longer than $10^{4} \mathrm{~s}$ and ON/OFF ratio higher than $10^{3}$. Many parameters about RS performance of RRAM devices were compared in this review, which can be also observed in Table 1.

\subsection{Thin Film Materials of Electrode}

Although materials application of RS medium has played a decisive role in performance of RRAM device, the effect of electrode thin film materials on devices cannot be neglected, which act as primary transport paths for carriers. For now, a great number of thin film materials have been investigated for the selection of electrode, such as elementary substance metals, carbon-based materials, conductive oxides, complex nitrides, and silicon-based materials. Table 2 demonstrated devices with various electrodes. During the research and selection of different materials for electrode of RRAM devices, some vital factors should be under special consideration, such as the activity of materials, work function of materials and the interface between electrode and RS layer [48]. 

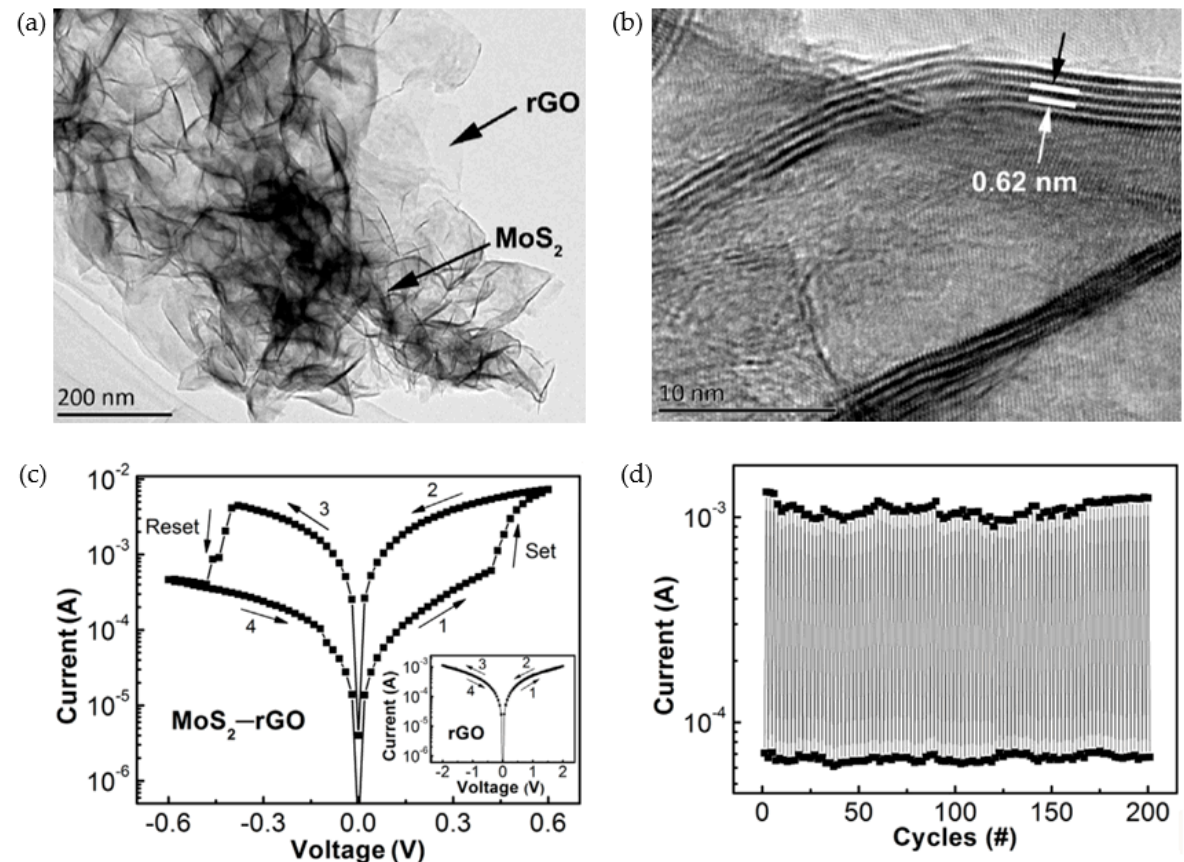

Figure 8. $\mathrm{MoS}_{2}$-rGO hybrid with (a) TEM and (b) HRTEM. (c) Bipolar I-V curves and (d) endurance properties of Ti/MoS 2 -rGO/ITO RRAM device, reproduced from [26], with permission from Elsevier, 2019.

Table 2. RRAM devices with various materials as electrodes.

\begin{tabular}{|c|c|c|c|c|c|c|c|}
\hline $\begin{array}{l}\text { Electrode } \\
\text { Materials }\end{array}$ & $\begin{array}{l}\text { Thickness } \\
\text { (nm) }\end{array}$ & $\begin{array}{l}\text { Electrode } \\
\text { Mode }\end{array}$ & $\begin{array}{c}\text { Switching } \\
\text { Mode }\end{array}$ & $\mathrm{V}_{\mathrm{SET}}(\mathrm{V})$ & $\mathrm{V}_{\text {RESET }}(\mathrm{V})$ & ON/OFF Ratio & Ref. \\
\hline Hf & $\sim 40$ & $\mathrm{TE}$ & Bipolar & $\sim 4$ & $\sim-4$ & $\sim 10^{3}$ & [129] \\
\hline $\mathrm{Al}$ & $\sim 40$ & $\mathrm{TE}$ & Bipolar & $\sim 2$ & $\sim-2$ & $>10^{2}$ & [27] \\
\hline $\mathrm{Ti}$ & $\sim 100$ & $\mathrm{TE}$ & Bipolar & $\sim 0.5$ & $\sim-1.5$ & $\sim 10^{3}$ & [8] \\
\hline $\mathrm{Zr}$ & $\sim 40$ & $\mathrm{TE}$ & Bipolar & $\sim 2$ & $\sim-4$ & $\sim 10^{3}$ & [129] \\
\hline $\mathrm{Cr}$ & $\sim 70$ & $\mathrm{TE}$ & Bipolar & $\sim 1.5$ & $\sim-1.5$ & $\sim 10^{4}$ & [130] \\
\hline $\mathrm{Ni}$ & $\sim 40$ & $\mathrm{TE}$ & Bipolar & $\sim 1.0$ & $\sim-1.0$ & $\sim 10^{3}$ & [9] \\
\hline $\mathrm{Cu}$ & $\sim 150$ & TE & Bipolar & $\sim 2.0$ & $\sim-2.0$ & $\sim 10^{3}$ & [10] \\
\hline $\mathrm{Ag}$ & $\sim 140$ & $\mathrm{TE} / \mathrm{BE}$ & Bipolar & $\sim 2.0$ & $\sim-2.0$ & $\sim 10$ & [11] \\
\hline $\mathrm{Pt}$ & $\sim 200$ & $\mathrm{BE}$ & Bipolar & $\sim 6.0$ & $\sim-5.0$ & $\sim 10^{3}$ & [12] \\
\hline $\mathrm{Au}$ & $\sim 50$ & $\mathrm{TE} / \mathrm{BE}$ & Bipolar & $\sim 1.0$ & $\sim-2.0$ & $\sim 10^{2}$ & [78] \\
\hline Graphene & N. A & TE & Unipolar & $\sim 1.0$ & $\sim-1.0$ & $\sim 10^{4}$ & [131] \\
\hline ITO & $\sim 50$ & $\mathrm{BE}$ & Bipolar & $\sim 1.5$ & $\sim-1.0$ & $\sim 10^{2}$ & [20] \\
\hline $\mathrm{TiN}$ & $\sim 20$ & $\mathrm{TE}$ & Bipolar & $\sim 0.5$ & $\sim-1.0$ & $\sim 10^{2}$ & [85] \\
\hline $\mathrm{TaN}$ & $\sim 60$ & $\mathrm{TE}$ & Bipolar & $\sim 1.5$ & $\sim-1.0$ & $\sim 10^{2}$ & [20] \\
\hline p-type Si & $\sim 100$ & $\mathrm{BE}$ & Bipolar & $\sim 2$ & $\sim-2$ & $>10^{2}$ & [27] \\
\hline
\end{tabular}

In general, the elementary substance metals are the most common and widely applied in RRAM devices, including Hf [129], Al [27], Ti [8], Zr [129], Cr [27], Ni [9], Cu [10], Ag [11], Pt [12], and Au [78]. Most of the time, RRAM devices always exhibit unipolar RS performance with noble metal electrode, such as Pt and Ru both as TE and BE $[48,88]$. However, the bipolar RS behavior will be always observed when one of the noble metal electrodes is replaced by an active metal electrode like $\mathrm{Ni}$ and $\mathrm{TiN}[8,85]$. Chen et al. reported a $\mathrm{Ta}_{2} \mathrm{O}_{5}$-based RRAM device with metal $\mathrm{Hf}$ as TE and they covered a 50-nm-thick Pt layer onto the Hf layer to prevent the oxidation [129]. The $\mathrm{Pt} / \mathrm{Hf} / \mathrm{Ta}_{2} \mathrm{O}_{5} / \mathrm{Pt}$ device operated under $\sim 4 \mathrm{~V}$ with ON/OFF ratio higher than $10^{3}$, which resulted from the fact that a higher Ta atomic concentration was observed at the $\mathrm{Hf} / \mathrm{Ta}_{2} \mathrm{O}_{5}$ interface than that at the $\mathrm{Ta}_{2} \mathrm{O}_{5} / \mathrm{Pt}$ interface. It is further indicated that considerable diffusion of oxygen ions occurred in the Hf electrode and the obvious degeneration of $\mathrm{O} / \mathrm{Ta}$ ratio occurred at the $\mathrm{Hf} / \mathrm{Ta}_{2} \mathrm{O}_{5}$ interface, which can be illustrated in Figure 9. A similar structure was also reported to replace $\mathrm{Hf}$ with $\mathrm{Zr}$ as $\mathrm{TE}$ in $\mathrm{Pt} / \mathrm{Zr} / \mathrm{Ta}_{2} \mathrm{O}_{5} / \mathrm{Pt}$ device, which showed lower operation voltage than that of the $\mathrm{Pt} / \mathrm{Hf} / \mathrm{Ta}_{2} \mathrm{O}_{5} / \mathrm{Pt}$ device. Shen et al. 
proposed a GO-based RRAM device with Al and p-type Si as TE and BE, respectively [27]. Their RRAM device with solution-processed GO dielectric layer exhibited excellent performance with SET/RESET voltage lower than $2 \mathrm{~V}$ and $\mathrm{ON} / \mathrm{OFF}$ ratio higher than $10^{2}$, which was affected by the diffusion of oxygen ions between metal $\mathrm{Al}$ and $\mathrm{GO}$ thin film. The $\mathrm{Cr} / \mathrm{Gd}_{2} \mathrm{O}_{3} / \mathrm{TiN}$ RRAM device was proposed by Jana et al. [130]. The bipolar RS characteristics demonstrated that the RRAM device could operate with operation voltage lower than $1.5 \mathrm{~V}$ and ON/OFF ratio higher than $10^{4}$. This 9-nm-thickness device exhibited excellent repeatable RS cycles with number of endurance pulses over $10^{5}$ and retention time longer than $3 \times 10^{4} \mathrm{~s}$. In addition, some RRAM devices with noble metal as both TE and BE also demonstrated bipolar switching performance, such as $\mathrm{Pt} /$ sputtering-deposited $\mathrm{Cr}_{2} \mathrm{O}_{3} / \mathrm{Pt}$ reported by Chen et al. [132], Pt/sputtering-deposited $\mathrm{TaO}_{\mathrm{x}} / \mathrm{Pt}$ reported by Huang et al. [133], Pt/Ni/ECD (electrochemical deposition)-fabricated $\mathrm{CuO}_{\mathrm{x}} / \mathrm{Ni} / \mathrm{Pt}$, and $\mathrm{Pt} /$ sputtering-deposited $\mathrm{SiO}_{2} / \mathrm{Pt}$ reported by Park et al. [134].

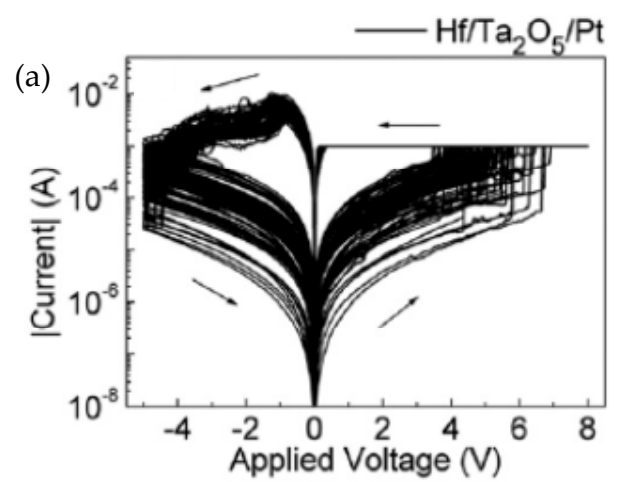

(b)

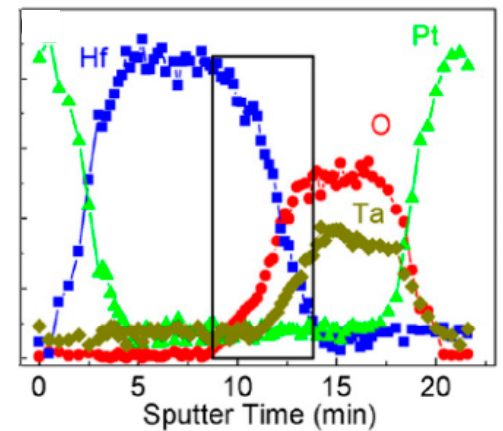

Figure 9. (a) Bipolar RS performance and (b) AES depth profiles of $\mathrm{Hf} / \mathrm{Ta}_{2} \mathrm{O}_{5} / \mathrm{Pt}$ RRAM devices, reproduced from [129], with permission from AIP Publishing, 2013.

Apart from elementary substance metals mentioned, carbon-based materials such as graphene and carbon nanotubes [48,62,131,135], conductive oxides such as ITO (indium tin oxide) [131], and complex nitrides such as TiN and TaN $[20,85]$ are also charming as selection of RRAM electrodes. ITO electrode has been widely utilized in electronic devices as well as RRAM cells due to its excellent electrical conductivity, high light transmittance and high hardness [48]. In addition, as one of the n-type semiconductors with highly degeneration characteristic, the ITO electrode has good electrical conductivity due to internal carriers including oxygen vacancy and activated $\mathrm{Sn}^{4+}$ ions [48]. Zhao et al. proposed an RRAM device with multilayer graphene (MLG) and ITO as TE and BE, respectively [131]. Their transparent MLG/ $\mathrm{Dy}_{2} \mathrm{O}_{3} / \mathrm{ITO}$ device exhibited $80 \%$ transmittance at $550 \mathrm{~nm}$ wavelength due to the good light transmittance of the ITO electrode. The excellent electrical conductivity of graphene and ITO made the devices operate under low CC $(<100 \mu \mathrm{A})$ with low voltage $(<1 \mathrm{~V})$ and low power consumption $(<100 \mu \mathrm{W})$. The device also demonstrated a resistance ratio between HRS and LRS higher than $10^{4}$ during 200 successful switching cycles and retention longer than $10^{4} \mathrm{~s}$.

The significance of selecting electrode materials is to optimize the performance of RRAM devices. Electrode materials like inert metals ( $\mathrm{Pt}$ and $\mathrm{Pd}$ ) are always investigated as carrier transportation paths, which have a slight and limited influence on RS performance. Then, electrodes of an RRAM device should have a positive impact on the formation process of CFs, which resulted from the migration of anion, which is always observed in oxygen-vacancy-based RRAM devices [8,9,32]. Finally, the selection of electrode materials for RS medium can directly affect the concentration of migrated anion and accumulated vacancy/cation; proper choice of electrode materials will have a positive influence in the formation process of CFs and also achieve stability enhancement of RRAM device. 


\section{Bionic Synaptic Application}

With the rapid development of information technology, the neuromorphic computing applications in terms of hardware and software have stepped into the new era. For now, the neuron complexity simulation has been achieved with conventional computing technology based on animal brains of cats and rats [136,137]. However, as illustrated in Figure 10, it has been considered to be inefficient to process huge amounts of data with traditional computing architecture like Von Neumann architecture. The Von Neumann architecture was a binary-based computer concept structure proposed by John von Neumann in 1946, which indicated that a program should be considered as a part of data firstly [136,137]. Based on this architecture, the program itself and the data it processes should be stored with the same memory technology. The memory address of program instruction and the memory address of data should point to different physical locations in the same memory device $[138,139]$. Besides, the width of program instruction should be the same as that of processed data. As illustrated in Figure 10a, programs in matrix multiplication always process a lot of data at a slow speed, and the speed is slowed down with the increase of hidden layers. Energy power consumption also increases at the same time. Compared with CPU and GPU-based computer systems, although emerging dedicated FPGA and ASIC hardware chips have demonstrated faster operation speed and lower energy consumption, the matrix multiplication for large amounts of data is still limited by operation speed and energy consumption.

(a)

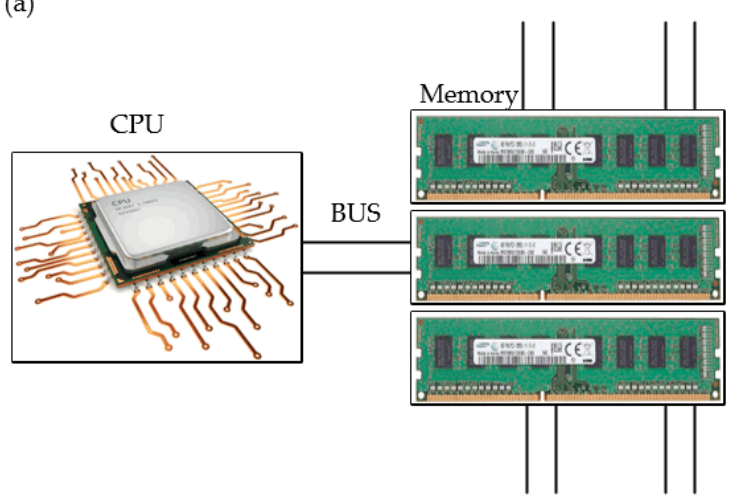

(b)

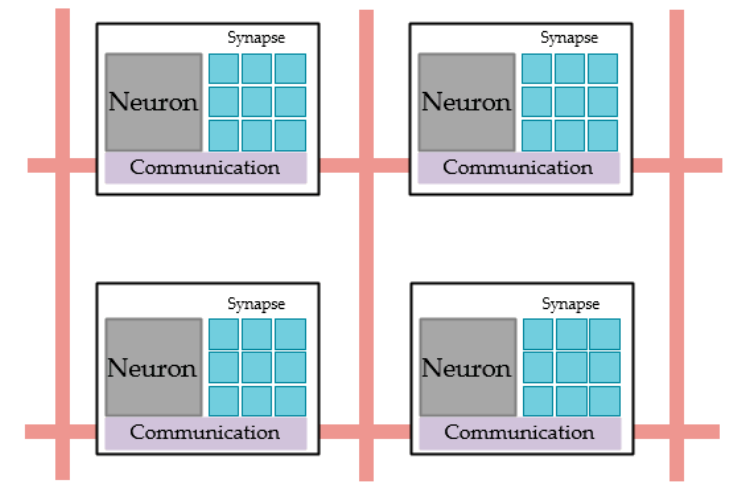

Figure 10. (a) Computing systems based on traditional Von Neumann architecture, the memory address of program instruction and the memory address of data point to different physical locations in the same memory device. (b) Computing systems based on neuromorphic architecture with integration of a single synaptic device into each unit.

Compared with a conventional computer system based on Von Neumann architecture, artificial neural networks (ANNs) have received extensive attention due to their lower power consumption and higher operation efficiency $[138,139]$. However, conventional synaptic devices cannot meet the requirements of ANNs because of some technological limitations like large device area, high power consumption and slow response speed. As illustrated in Figure 10b, emerging synaptic device can be integrated in to a single unit and improves the processing efficiency and processing accuracy $[15,17,34-38]$. The superior RS characteristics demonstrated in RRAM devices reveal its great potential in the neuromorphic application based on ANNs. An RRAM device can store multi-bit information in a non-volatile manner on a $4 \mathrm{~F}^{2}$ device area and can also switch with the energy of $\sim$ pJ-level, which enables significant improvements in high-density integration and low power operation compared to conventional CMOS-based synapse devices [135-138].

In the analog circuit of ANNs, RRAM devices act as substitution to synapses in order to provide connection function between neurons and information storage cells [140,141]. The ultra-small size of RRAM device is to increase the synapse density of ANNs, which is expected to reach the synapse density in human brain $\left(\sim 10^{10}\right.$ synapses/ $\left.\mathrm{cm}^{2}\right)[28,142]$. As the key role during the information delivery process, a synapse is responsible for transmitting impulses from one neuron to another, which is considered to 
provide dynamical interconnections between two connecting neurons. Figure 11 demonstrated the basic structure of biological synapse, which mainly includes presynaptic membrane, synaptic cleft, postsynaptic membrane, and neurotransmitter [143]. Presynaptic membrane and synaptic vesicle are included by the presynaptic element, which is the dendrite/axon terminal of pre-neuron. Chemical substances in synaptic vesicle are called neurotransmitters. With the external spiking or stimulation onto the presynaptic element, synaptic vesicle filled with neurotransmitter will be released from presynaptic membrane to postsynaptic membrane due to the flux of $\mathrm{Ca}^{2+}$, which has a temporary influence on synaptic transmission. The transmitting process of the neurotransmitter through a synapse represents the information delivery among neurons. The gap between presynaptic and postsynaptic membranes is the synaptic cleft, which is around 15 30-nm thick [144,145]. In general, for application in neuromorphic system and ANNs, capacitor-like synaptic devices are always used to simulate neuron bodies in the human brain; a neuron in the human brain can be compared to an electronic device with combined functions including an integrator and a device for threshold spiking. An axon acts like a connecting bridge in order to provide a transmission connection of information, which can be considered as a long wire. A dendrite works to transmit the signal input from multiple neurons to a single neuron, which can be compared to a short wire. A synapse acts as a connector to provide connection function between two next neurons [2], which have been attached to the most interests for now.

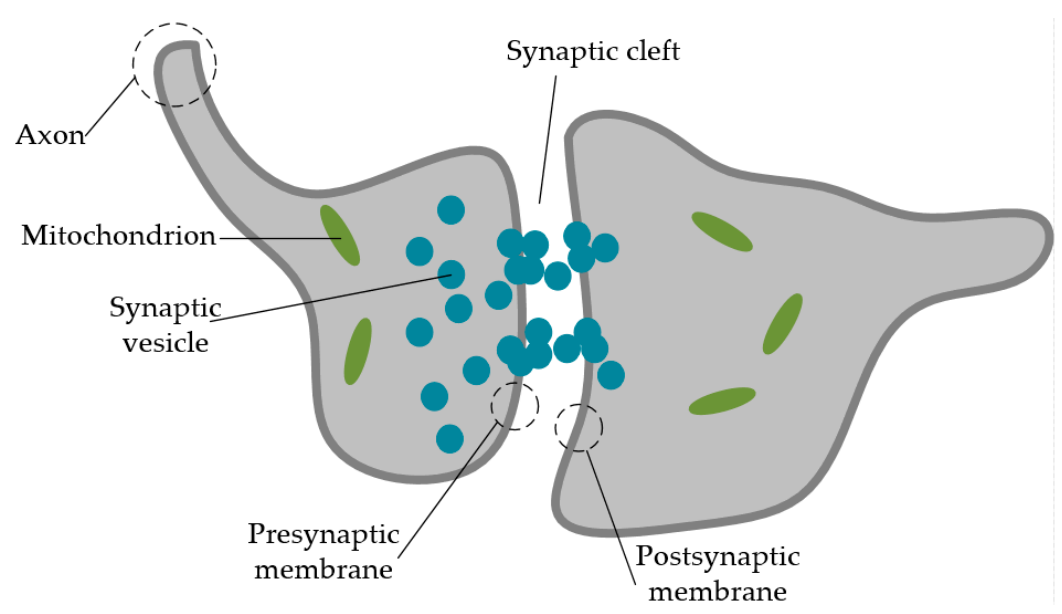

Figure 11. Structure of synapse in neural network.

Massive neurons form neural circuits through synapses in neural networks of the human brain [1-6]. These neural circuits are the foundation of advanced neural activities such as learning and memorizing, which are attributed to the plasticity of neural networks [2-4]. Basically, the plasticity of neural network is corresponding to the synaptic plasticity, which is dependent on synaptic weight [146,147]. Synaptic weight is used to demonstrate the strength or amplitude of connection between the two next neurons, which is mainly related to the amount of released or absorbed neurotransmitter. According to the theory proposed by Hebbian in 1949 [148], the connection between the two next neurons can be strengthened when they receive stimulation signals, which will result in the potentiation and depression during the neuron activities. For instance, long-term potentiation is corresponding to the synaptic potentiation behavior while long-term depression indicates depression performance of synapse. The synaptic plasticity mainly induced by the change of synaptic weight is divided into long-term plasticity (LTP) and short-term plasticity (STP) $[149,150]$. STP includes short-term potentiation, pair-pulse facilitation (PPF) and depression while long-term potentiation and depression are considered as representations of LTP [151]. For a biological neural system, on the one hand, STP is responsible for critical computation, on the other hand, LTP is thought of as the foundation of ability in terms of machine learning and memorizing [151]. As the molecular mechanism for learning and memorizing in the human brain, the timescale of STP can sustain from tens of milliseconds to few minutes, while the retention time of 
LTP can be over a few hours, even several days. The transition from STP to LTP can be achieved through repeated stimulation during the learning process. Apart from LTP and STP, the plasticity depends on spike time (spike-time-dependent plasticity, STDP) is also under investigation, which is one of the advanced learning characteristics in the neuron system of the human brain [152,153]. As indicated by Hebb's rule, when the presynaptic membrane is stimulated earlier than the postsynaptic membrane and the postsynaptic current can be enhanced, which is called long-term potentiation. Reversely, when the spike occurs on the postsynaptic membrane earlier than that on the presynaptic membrane, the postsynaptic current will be depressed, namely long-term depression. In addition, it is noted that with the change of relative time between two stimulations (commonly pulses), the postsynaptic current will also be influenced [154]. Due to the existence of RS characteristics, the resistance states of RRAM devices can be manipulated by applied voltages and the microscopic conductive paths through the RS medium will be acquired. Therefore, resistance values of RRAM devices are the response to periodic input signals (voltage or current), which can be considered as the synaptic weights. The modification operations of resistance represent the changes of synaptic weights. When the external power supply fails to work, resistance states can also be retained [2].

\subsection{Short-Term Plasticity for RRAM Devices}

In general, two types of RS behaviors observed in RRAM devices, abrupt and gradual RS behaviors, were considered corresponding to digital and analog switching, respectively. The abrupt performance for resistance change was believed to be consistent with a digital signal while the gradual one with continuous conductance changes showed similar characteristics like a biological synapse [155]. Ilyas et al. demonstrated their research on a physical-vapor-deposited $\mathrm{Ag} / \mathrm{SiO}$ : $\mathrm{Ag} / \mathrm{TiO} / \mathrm{p}^{++}-\mathrm{Si}$ RRAM device with bilayer dielectric in terms of STP such as potentiation, depression and PPF [155]. The conductance of this bilayer device was modified by positive and negative pulses. As illustrated in Figure 12a,b, with the repeated voltage sweep, the potentiation and depression behaviors were observed and indicated the conductance states change during the processes of potentiation and depression, which proved the feasibility of conductance modification and simulated the change of synaptic weight. Figure 12c demonstrated the results of PPF for this bilayer device. PPF is always investigated to provide adjustment for conductance in order to perform short-term neural behaviors such as synaptic filtering and adaptation [156,157]. During two continuous pulse stimulations of their $\mathrm{Ag} / \mathrm{SiO}_{\mathrm{x}}: \mathrm{Ag} / \mathrm{TiO}_{\mathrm{x}} / \mathrm{p}^{++}-\mathrm{Si}$ samples, the next post-synaptic response became higher than that of the previous one, which indicated that the interval time of spike was less than the recovery time. Berdan et al. also reported the STP of $\mathrm{TiO}_{2}$-based RRAM devices through the transient conductance response [158]. As illustrated in Figure 12d, the conductance of the device increased due to the applied pulse and then decayed back to its initial state slowly. A similar performance could be observed on subsequent pulses who were dependent on the previous resistance states. Apart from RRAM devices with traditional metal oxides, short-term synaptic performance of devices fabricated with $2 \mathrm{D}$ materials are also under investigation [159-161]. Sun et al. reported their research on 2D-material-based devices with h-BN as a functional layer [159]. As demonstrated in Figure 12e, an obvious increase could be observed for ON-state current, which indicated the rise of synaptic strength due to the repeated pulse stimulation. The short-term facilitation with applied voltage-pulse excitation was observed on $\mathrm{Ag} / \mathrm{h}-\mathrm{BN} / \mathrm{graphene}$ device as well. Sokolov et al. also provided their investigation results about PPF response of RRAM devices based on $\mathrm{TaO}_{x} / \mathrm{IGZO}(\mathrm{InGaZnO})$ bilayers [162]. Firstly, the single pulse with $0.75 \mathrm{~V}$ amplitude and $2 \mathrm{~ms}$ width was applied onto TE of RRAM device and the device exhibited a corresponding current response of $\sim 220 \mathrm{~mA}$. After that, with the RESET operation, the device transferred to HRS with initial OFF-state current. Then, two consecutive stimuli equal in amplitude and width to the first single pulse were applied onto TE, and the current response was $~ 350 \mathrm{~mA}$, which indicated that the $\mathrm{TaO}_{\mathrm{x}} / \mathrm{IGZO}$-based RRAM device could enhance the trans-conductance with the effect of high-frequency stimuli and further improve the PPF phenomenon. 

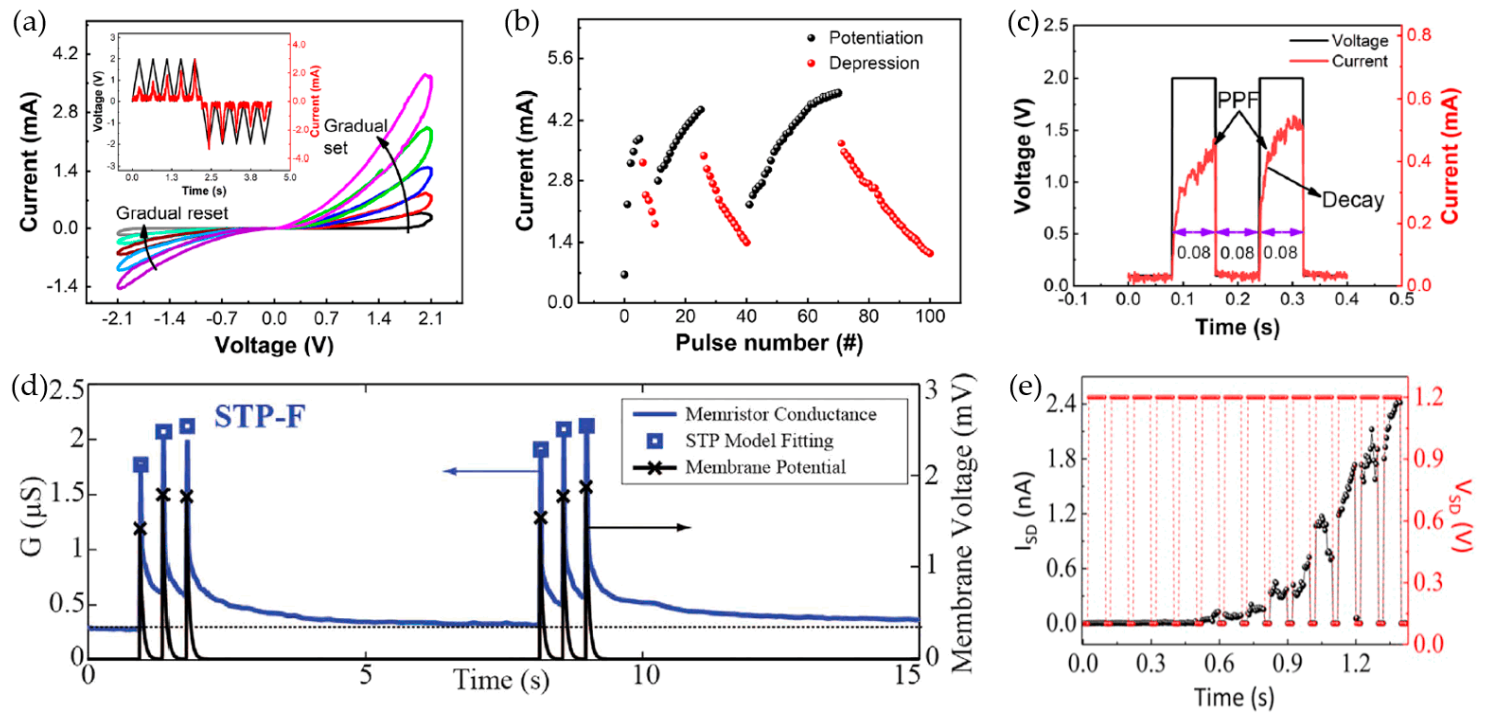

Figure 12. (a) Potentiation and depression response of $\mathrm{Ag} / \mathrm{SiO} \mathrm{x}_{\mathrm{x}}: \mathrm{Ag} / \mathrm{TiO}_{\mathrm{x}} / \mathrm{p}^{++}-\mathrm{Si}$ device with repeated voltage sweeps. (b) Conductance modulation and (c) PPF of $\mathrm{Ag} / \mathrm{SiO}{ }_{\mathrm{x}}: \mathrm{Ag} / \mathrm{TiO} \mathrm{x}_{\mathrm{x}} / \mathrm{p}^{++}-\mathrm{Si}$ device by repeating consecutive pulses. (d) Repeated STP response with the model fitting of $\mathrm{TiO}_{\mathrm{x}}$-based RRAM device, reproduced from [158], with permission from Springer Nature, 2020. (e) Synaptic facilitation response to consecutive pulses of the device with h-BN, reproduced from [159], with permission from Elsevier, 2020.

\subsection{Long-Term Plasticity for RRAM Devices}

Compared with the investigation of STP, current research on LTP mainly focuses on long-term potentiation/depression and transition from STP to LTP $[79,163,164]$. Wang et al. proposed a flexible bipolar RRAM device with ALD-deposited $\mathrm{Hf}_{0.5} \mathrm{Zr}_{0.5} \mathrm{O}_{2}(\mathrm{HZO})$ dielectric layer, as illustrated in Figure 13a-c, which worked as artificial synapses in the neuromorphic network in order to overcome the bottleneck based on traditional Von Neumann structure [79]. During the 400 continuous programming pulses, devices with gradual RS behaviors in DC sweep exhibited the feasibility of conductance modification under a sequence of consecutive programmable pulses, which indicated that the synaptic devices that emulated long-term potentiation/depression had great potential of artificial application in a neuromorphic computing system. The potentiated and depressed performance were stimulated by applying $2000.8 \mathrm{~V} / 20 \mathrm{~ms}$ continuous pulses and $2000.5 \mathrm{~V} / 20 \mathrm{~ms}$ continuous pulses in Figure 13a, respectively. This Ag/HZO/ITO/PET RRAM device showed the decayed post-synaptic current (PSC), and the PSC state turned into intermediate over time due to the forgetting effect. Figure 13b,c showed excitatory and inhibitory states of PSC with a single pre-synaptic spike, and the retention time over 1000s demonstrated the reliability of STP in Ag/HZO/ITO/PET RRAM devices. Wang et al. demonstrated their research report on a flexible RRAM device fabricated with a common polymer, poly (3,4-ethylene dioxythiophene): poly (styrene sulfonate) (PEDOT:PSS), as functional layer [163], and the PEDOT:PSS solution was spin-coated onto the ITO substrate. They chose consecutive positive and negative voltage bias carefully to apply onto TE Au of their device in order to avoid abrupt RS performance. The decreased conductance was observed when the continuous positive voltage bias swept from $0 \mathrm{~V} \rightarrow 3 \mathrm{~V} \rightarrow 0 \mathrm{~V}$ and then the gradual increased conductance was demonstrated with the consecutive negative voltage bias from $0 \mathrm{~V} \rightarrow-2 \mathrm{~V} \rightarrow 0 \mathrm{~V}$, which indicated the potential of successful modulation of synaptic weights. The consecutive pulses with $2 \mathrm{~V}$ amplitude and $10 \mathrm{~ms}$ width were applied to TE for observing the current response, as demonstrated in Figure 13d; the potentiation and depression responses were observed with five consecutive positive pulses bias and five consecutive negative pulses bias, respectively. After that, the Au/PEDOT:PSS/ITO RRAM device was applied consecutive pulses (300 1V/10 ms positive pulses and $300-1.5 \mathrm{~V} / 10 \mathrm{~ms}$ negative pulses) and the long-term potentiation and depression were emulated successfully in Figure 13e. Finally, Wang et al. 
repeated the pulse trains five times, as illustrated in Figure 13f, which indicated the excellent endurance property of their device.
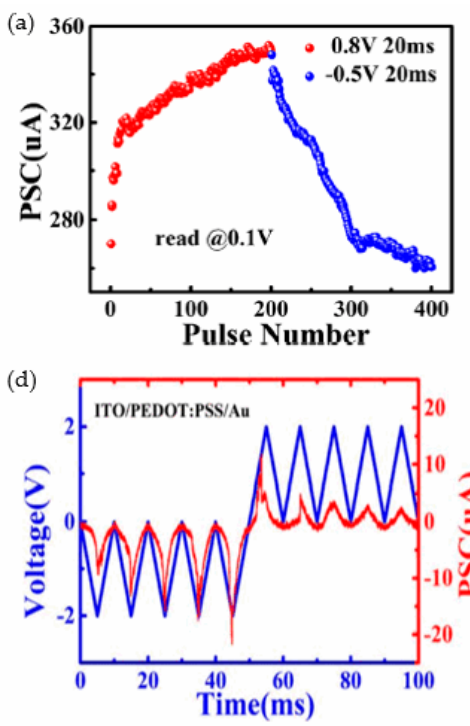
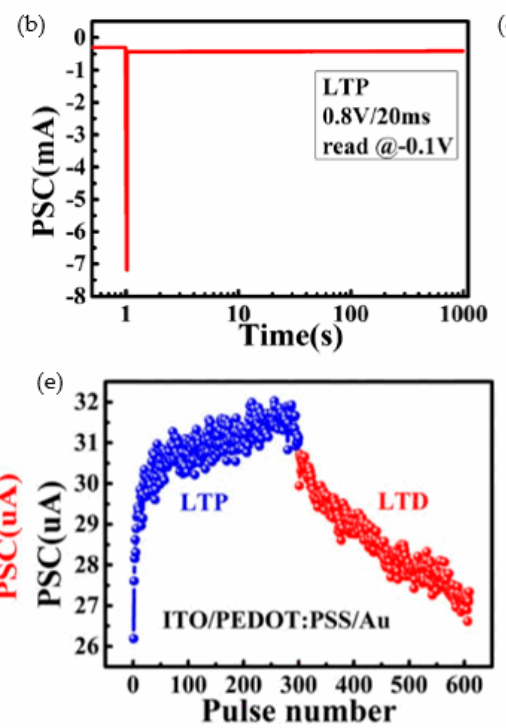
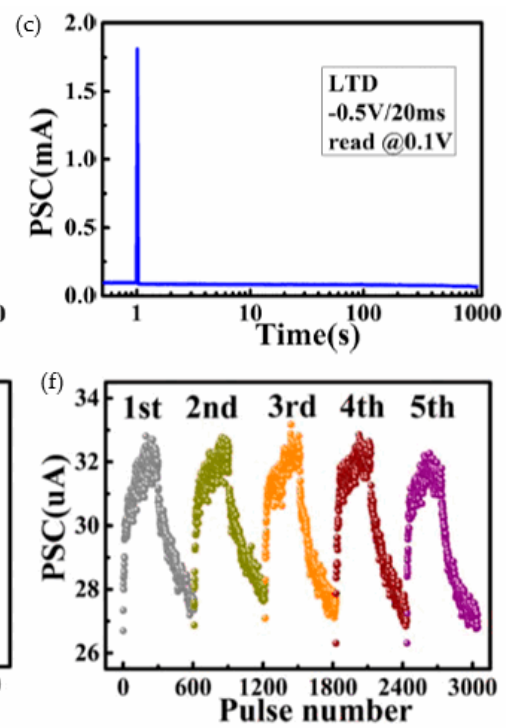

Figure 13. (a) Gradual modulation for conductance with long-term potentiation/depression response of Ag/HZO/ITO/PET RRAM device. Retention performance of Ag/HZO/ITO/PET RRAM device in (b) long-term potentiation process by consecutive positive pulses and (c) long-term depression process by consecutive negative pulses, reproduced from [79], with permission from Springer Nature, 2019. (d) Synaptic weights modulation of Au/PEDOT:PSS/ITO RRAM device by 10 consecutive pulses. (e) Long-term potentiation/depression under 600 consecutive pulses in one operation and (f) five operations of long-term potentiation/depression for Au/PEDOT:PSS/ITO RRAM device, reproduced from [163], with permission from MDPI (Basel, Switzerland), 2018.

Except for the research on long-term potentiation and depression behaviors of RRAM devices, the transition from STP to LTP also attracted significant interests [151,155,165], which is corresponding to transition from short-term memory (STM) to long-term memory (LTM). Biologically, compared with LTP, the sustainment time of STP is shorter generally, which is related to forgetting behaviors in the human brain. However, the STP can transfer into LTP with the repeated stimuli or a series rehearsal operation [155]. Zhang et al. reported the mechanism of conversion from STM to LTM in Cu/a-Si/Pt RRAM device [151]. As illustrated in Figure 14a, there were little $\mathrm{Cu}^{2+}$ ions drifting into the RS layer with a few stimulations, and then CF based on $\mathrm{Cu}$ atoms spontaneously decayed back to the initial state. However, with more repeated stimulations onto TE $\mathrm{Cu}$, more $\mathrm{Cu}^{2+}$ ions migrated to the RS layer, which showed similar performance like training operation in the neural network. Ilyas researched the transition from STP to LTP of $\mathrm{Ag} / \mathrm{SiO}_{\mathrm{x}}: \mathrm{Ag} / \mathrm{TiO}_{\mathrm{x}} / \mathrm{p}++-\mathrm{Si}$ device through modulating pulse strength [155]. Figure $14 \mathrm{~b}, \mathrm{c}$ showed the normalized current response by applying pulses with various amplitudes $(1.2 \mathrm{~V}, 1.8 \mathrm{~V}, 2.0 \mathrm{~V}$ and $2.8 \mathrm{~V})$ and each training cycle included 50 pulses. With the experimental results and the fitting results based on the equation in Figure $14 \mathrm{~b}$, the increased tendency of relaxation time was confirmed due to the effect of pulse strength (the red line in Figure 14c). An obvious elevation of synaptic weight was also observed when the relaxation time was around $45 \mathrm{~s}$ and the pulse amplitude was $+2.8 \mathrm{~V}$, which indicated that STP has transferred to LTP. 
(a)

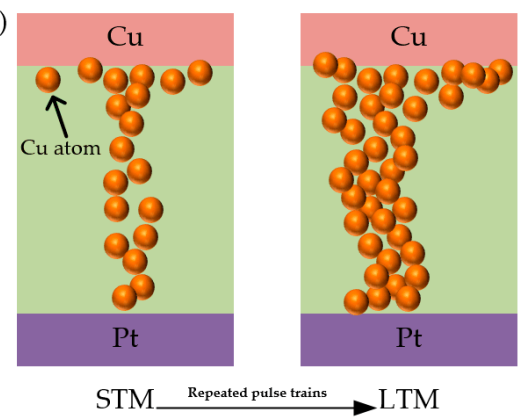

(b)

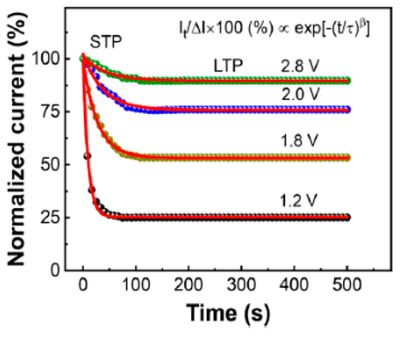

(c)

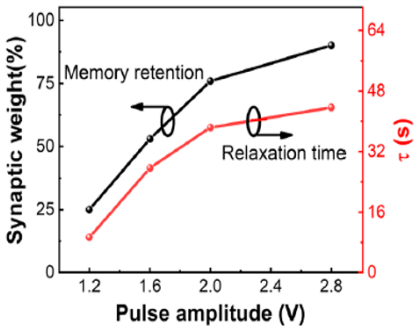

Figure 14. (a) Illustration of $\mathrm{Cu}$ atom dynamics of $\mathrm{Cu} / \mathrm{a}-\mathrm{Si} / \mathrm{Pt}$ device during the transition from STM to LTM. (b) Relationship between normalized current response and retention time when the transferring process from STP to LTP occurred in $\mathrm{Ag} / \mathrm{SiO}_{\mathrm{x}}: \mathrm{Ag} / \mathrm{TiO}_{\mathrm{x}} / \mathrm{p}^{++}-\mathrm{Si}$ device. (c) Synaptic weight response to changes of pulse amplitude and relaxation time $\tau$, reproduced from [155], with permission from Springer Nature, 2020.

\subsection{Spike-Time-Dependent Plasticity for RRAM Devices}

Currently, many research achievements have proved that long- and short-term plasticity functions of biological neural synapse could by mimicked by RRAM devices with modulation of applied pulses (including amplitude and number modulations). On the other hand, as one of the determining factors in synapse plasticity, STDP also has received extensive attention. STPD can be defined as a function relationship between change of synaptic weight $(\Delta W)$ and time interval $(\Delta t)$ resulting from activity variation of the pre- and post-neurons $[155,163]$. When $\Delta t>0$, the activity of pre-synaptic neuron precedes that of post-synaptic neuron, which means that the connection strength between these two neurons will be reinforced and result in the long-term potentiation of the synapse. Conversely, the long-term depression occurs when the spike of post-synaptic neuron heads that of pre-synaptic neuron and the connection strength is weakened $(\Delta t<0)$. Obviously, for an RRAM device, top and bottom electrodes are compared to pre- and post-synaptic neurons and pulses applied onto electrode can mimic spikes of biological synapses. The polarity of $\Delta \mathrm{W}$ is determined by the order of spikes of pre- and post-synaptic neurons. In general, the time interval and change of synaptic weight are defined as follows:

$$
\begin{gathered}
\Delta t=t_{\text {post }}-t_{\text {pre }} \\
\Delta W=\frac{G_{t}-G_{i}}{G_{i}} \times 100 \%
\end{gathered}
$$

in which $t_{\text {pre }}$ and $t_{\text {post }}$ are time nodes of spikes applied onto pre- and post-synaptic neurons, respectively. $G_{i}$ is the conductance of the device at the initial state when $t$ is 0 , and $G_{t}$ is the conductance when the time reaches node $t$.

Ilyas et al. emulated the STDP rule of $\mathrm{Ag} / \mathrm{SiO}$ : $\mathrm{Ag} / \mathrm{TiO}_{\mathrm{x}} / \mathrm{p}^{++}-\mathrm{Si}$ samples, which can be observed in Figure 15a,b [155]. Through the implementation of a pair of pulses $\pm 1.2 \mathrm{~V} / 5 \mathrm{~ms}, \Delta \mathrm{W}$ decreased with the increase of $\Delta t$, which indicated that a more obvious conductance change could be observed when the time interval decreased. When $\Delta t>0$, the pre-spike occurred before the post-spike, and the increased $\Delta \mathrm{W}$ proved the enhancement of device conductance along with the decreasing $\Delta t$. Inversely $(\Delta t>0)$, the depression of device conductance was observed in $\Delta \mathrm{W}$ when $\Delta \mathrm{t}$ increased. Mahata et al. revealed the STDP characteristic of RRAM devices with bilayer metal-oxide dielectric $\left(\mathrm{TaN} / \mathrm{HfO}_{2} / \mathrm{Al}_{2} \mathrm{O}_{3} / \mathrm{ITO}\right)$ [20]. As illustrated in Figure 15c,d, a series of pulses with different amplitudes were applied on to TaN and ITO electrodes. The largest values of $\Delta \mathrm{W}$ were $97 \%$ and $-84 \%$ when the $|\Delta t|$ was $10 \mu$ s at both states $(\Delta t>0$ and $\Delta t<0)$, which confirmed that their bilayer RRAM devices had STDP behaviors at various spiking timings. Wang et al. reported the STDP behaviors of the RRAM device fabricated with organic 2D materials [163]. After the test of long-term potentiation and depression with 600 consecutive programming pulses, they used a pair of pulse $( \pm 1.5 \mathrm{~V} / 10 \mathrm{~ms})$ to provide spikes on TE and BE of 
device, and the related result can be observed in Figure 15e. When the pre-synaptic neuron was spiked earlier than the post-synaptic neuron, the potentiated response of connection strength between two neighboring neurons could be demonstrated. On the contrary, the decreased synaptic weight indicated the weak connection. Wan also reported the STDP of 2D-material-based RRAM with the structure of $\mathrm{Ag} / \mathrm{SrTiO}_{3} / \mathrm{RGO} / \mathrm{FTO}$ [166]; a similar experimental response with applied pulses ( $\left.\pm 1 \mathrm{~V} / 10 \mathrm{~ms}\right)$ was observed in Figure 15f. The relationship between time interval and change of synaptic weight was similar to results proposed by others, which was more similar to their fitting results. These results proved that a more considerable change of synaptic weight could be realized with the smaller time interval of activity between two adjacent synaptic neurons.

(a)

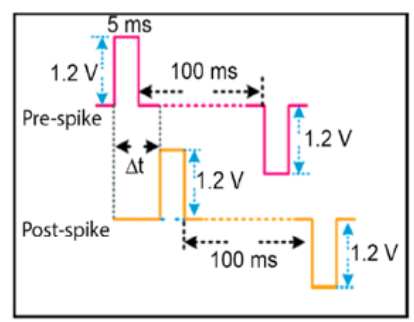

(d)

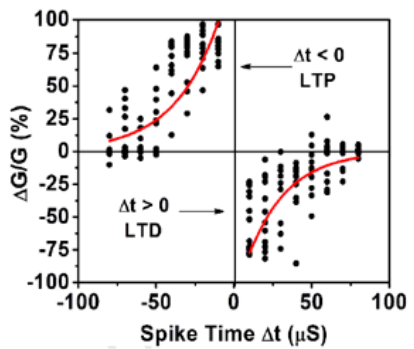

(b)

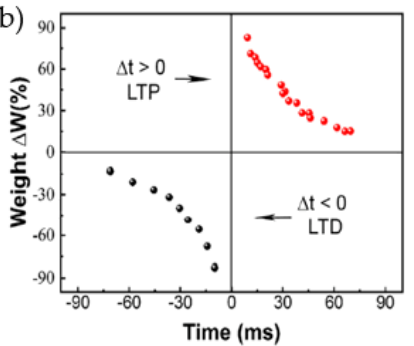

(e)

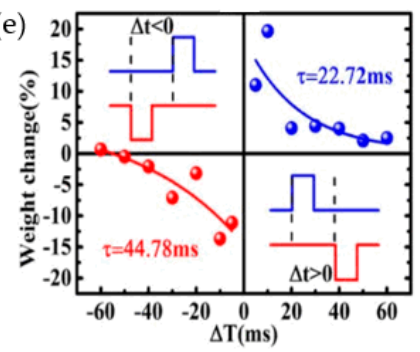

(c)

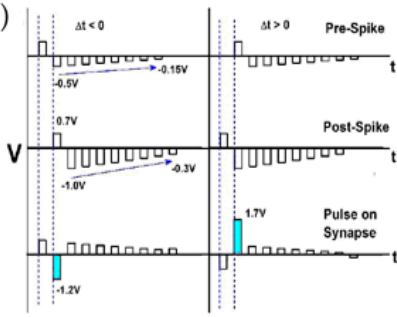

(f)

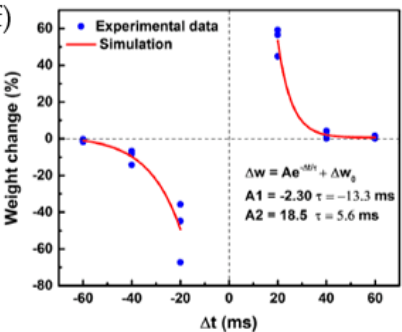

Figure 15. (a) Implementing programming pulses and (b) STDP behavior of $\mathrm{Ag} / \mathrm{SiO} \mathrm{x}_{\mathrm{x}}: \mathrm{Ag} / \mathrm{TiO} \mathrm{x}_{\mathrm{x}} / \mathrm{p}^{++}-\mathrm{Si}$ RRAM device, reproduced from [155], with permission from Springer Nature, 2020. (c) Applied pre/post-spikes with sequences and (d) STDP characteristics of TaN/ $\mathrm{HfO}_{2} / \mathrm{Al}_{2} \mathrm{O}_{3} / \mathrm{ITO}$ RRAM device, reproduced from [20], with permission from Elsevier, 2020. (e) STDP results with pulse interval modulation of pre- and post-synaptic spiking for PEDOT:PSS-based RRAM device, reproduced from [163], with permission from MDPI (Basel, Switzerland), 2018. (f) Experimental and fitting results of STDP behaviors for $\mathrm{Ag} / \mathrm{SrTiO}_{3} / \mathrm{RGO} / \mathrm{FTO}$ RRAM device, reproduced from [166], with permission from Elsevier, 2018.

\section{Conclusions}

In this work, we have provided an overview of RRAM devices with advances including various thin film materials applied in RS layer and electrode, classification of RS mechanisms and investigation on artificial synapse. Many research reports indicate that RRAM devices fabricated with inorganic materials, such as oxides, solid electrolyte and two-dimensional (2D) materials, have demonstrated relatively mature performance. There is great potential for the application of organic materials (biological and polymer materials) in RRAM devices accordingly. The performance of the devices depends largely on the RS mechanisms, which also has a strong connection with choice and processing techniques of the thin film materials. Based on the fundamental performance of RRAM devices, some outstanding enterprise or research institutions such as Samsung Electronics, Intel Corporation and Institute of Microelectronics of the Chinese Academy of Sciences (IMECAS) have put effort into promoting the development of large-scale manufacture and mature product commercialization of RRAM devices for many years. As early in 2004, Samsung Electronics reported the highly scalable TMO (binary transition-metal-oxide) RRAM devices with CMOS technology in IEDM (International Electron Devices Meeting) and $\mathrm{NiO}$ was used as a functional layer [167]. In 2007, the 2-MB CBRAM test chip was reported by Infineon and Samsung exhibited the 3D RRAM array with 1D1R structure [2]. 
From 2010 to 2013, Unity Semiconductor Corporation firstly reported their 64-MB RRAM test chip and SanDisk/Toshiba reported their 32-Gbit bilayer RRAM test chip. They realized the practical experiment in electrical circuits with devices fabricated by $\mathrm{TaO}_{x}$ and $\mathrm{HfO}_{x}$ functional layers [168]. One year later, Micron/Sony presented their 27-nm 16-Gbit CBRRAM test chip and $\mathrm{TaO}_{\mathrm{x}} / \mathrm{HfO}_{\mathrm{x}}$ functional layers with stack structure were investigated [169]. In 2016, the four-layer 3D vertical RRAM array with self-selecting characteristic was reported by IMECAS, and RS performance of $\mathrm{HfO}_{\mathrm{x}}$ resistive layers with the multi-level structure was verified [170]. In 2019, Intel Corporation announced they had prepared to manufacture emerging RRAM devices with $22 \mathrm{~nm}$ process technology, which also indicated that binary metal oxides or perovskite materials might be considered as candidates for the selection of functional layers [171]. All these developments are proving that the potential of large-scale commercialization for RRAM technology with different materials (especially TMO) is enormous and promising. Apart from the traditional large-scale commercialization process, the final objective of investigating different RRAM device performances is to provide potential assistance to artificial intelligence and neuromorphic computing systems. RRAM devices can mimic functions of biological synapse with electrical performance, which has a positive influence in hardware application of the artificial intelligence field. In addition, its human-brain-like behaviors such as STM and LTM make the development of neuromorphic computing system possible in the coming future.

Author Contributions: Conceptualization, Z.S. and C.Z. (Chun Zhao); Methodology, Z.S., C.Z. (Chun Zhao) and Y.Q.; Software, C.Z. (Chun Zhao), C.Z. (Cezhou Zhao) and L.Y.; Validation, C.Z. (Chun Zhao) and C.Z. (Cezhou Zhao); Formal Analysis, Z.S. and C.Z. (Chun Zhao); Investigation, Z.S., C.Z. (Chun Zhao), W.X. and Y.Q.; Resources, Z.S. and C.Z. (Chun Zhao); Data Curation, Z.S.; Writing-Original Draft Preparation, Z.S.; Writing-Review \& Editing, Z.S., C.Z. (Chun Zhao), Y.L., L.Y., C.Z. (Cezhou Zhao) and I.Z.M.; Visualization, Z.S. and C.Z. (Chun Zhao); Supervision, C.Z. (Chun Zhao), C.Z. (Cezhou Zhao), I.Z.M., Y.L., W.X. and L.Y.; Project Administration, C.Z. (Chun Zhao) and C.Z. (Cezhou Zhao); Funding Acquisition, C.Z. (Chun Zhao), C.Z. (Cezhou Zhao) and I.Z.M. All authors have read and agreed to the published version of the manuscript.

Funding: This research was funded in part by the Natural Science Foundation of the Jiangsu Higher Education Institutions of China Program (19KJB510059), the Suzhou Science and Technology Development Planning Project: Key Industrial Technology Innovation (SYG201924), and the Key Program Special Fund in XJTLU (KSF-P-02, KSF-T-03, KSF-A-04, KSF-A-05, KSF-A-07).

Acknowledgments: The author Ivona Z. Mitrovic acknowledges the British Council UKIERI project no. IND/CONT/G/17-18/18.

Conflicts of Interest: The authors declare no conflict of interest.

\section{References}

1. Lv, Z.; Zhou, Y.; Han, S.-T.; Roy, V.A.L. From biomaterial-based data storage to bio-inspired artificial synapse. Mater. Today 2018, 21, 537-552. [CrossRef]

2. Hong, X.; Loy, D.J.; Dananjaya, P.A.; Tan, F.; Ng, C.; Lew, W. Oxide-based RRAM materials for neuromorphic computing. J. Mater. Sci. 2018, 53, 8720-8746. [CrossRef]

3. Zakaria, O.; Madi, M.; Kasugai, S. Introduction of a novel guided bone regeneration memory shape based device. J. Biomed. Mater. Res. B Appl. Biomater. 2020, 108, 460-467. [CrossRef] [PubMed]

4. Mullani, N.; Ali, I.; Dongale, T.D.; Kim, G.H.; Choi, B.J.; Basit, M.A.; Park, T.J. Improved resistive switching behavior of multiwalled carbon nanotube/ $\mathrm{TiO}_{2}$ nanorods composite film by increased oxygen vacancy reservoir. Mater. Sci. Semicond. Process. 2020, 108, 104907-104916. [CrossRef]

5. Du, X.; Tadrous, J.; Sabharwal, A. Sequential Beamforming for Multiuser MIMO With Full-Duplex Training. IEEE Trans. Wirel. Commun. 2016, 15, 8551-8564. [CrossRef]

6. Jamkhande, P.G.; Ghule, N.W.; Bamer, A.H.; Kalaskar, M.G. Metal nanoparticles synthesis: An overview on methods of preparation, advantages and disadvantages, and applications. J. Drug Deliv. Sci. Technol. 2019, 53, 101174-101184. [CrossRef]

7. Simmons, J.G.; Verderber, R.R. New conduction and reversible memory phenomena in thin insulating films. Proc. R. Soc. Lond. Ser. A Math. Phys. Sci. 1997, 301, 77-102.

8. Kang, X.; Guo, J.; Gao, Y.; Ren, S.; Chen, W.; Zhao, X. NiO-based resistive memory devices with highly improved uniformity boosted by ionic liquid pre-treatment. Appl. Surf. Sci. 2019, 480, 57-62. [CrossRef] 
9. Shen, Z.; Qi, Y.; Mitrovic, I.Z.; Zhao, C.; Hall, S.; Yang, L.; Luo, T.; Huang, Y.; Zhao, C. Effect of Annealing Temperature for Ni/AlOx/Pt RRAM Devices Fabricated with Solution-Based Dielectric. Micromachines 2019, 10, 446. [CrossRef] [PubMed]

10. Wang, Y.; Liu, H.; Wang, X.; Zhao, L. Impacts of Cu-Doping on the Performance of La-Based RRAM Devices. Nanoscale Res. Lett. 2019, 14, 22401-22409. [CrossRef] [PubMed]

11. Liang, D.; Li, X.; Wang, J.; Wu, L.; Chen, P. Light-controlled resistive switching characteristics in $\mathrm{ZnO} / \mathrm{BiFeO} / \mathrm{ZnO}$ thin film. Solid State Electron. 2018, 145, 46-48. [CrossRef]

12. Wang, G.; Hu, L.; Xia, Y.; Li, Q.; Xu, Q. Resistive switching in FeNi/Al2O3/NiO/Pt structure with various Al2O3 layer thicknesses. J. Magn. Magn. Mater. 2020, 493, 165728-165734. [CrossRef]

13. Patel, K.; Cottom, J.; Bosman, M.; Kenyon, A.J.; Shluger, A.L. An oxygen vacancy mediated Ag reduction and nucleation mechanism in SiO2 RRAM devices. Microelectron. Reliab. 2019, 98, 144-152. [CrossRef]

14. Li, L.; Chang, K.C.; Ye, C.; Lin, X.; Zhang, R.; Xu, Z.; Zhou, Y.; Xiong, W.; Kuo, T.P. An indirect way to achieve comprehensive performance improvement of resistive memory: When hafnium meets ITO in an electrode. Nanoscale 2020, 12, 3267-3272. [CrossRef] [PubMed]

15. Teherani, F.H.; Look, D.C.; Rogers, D.J.; Prezioso, M.; Merrikh-Bayat, F.; Chakrabarti, B.; Strukov, D. RRAM-based hardware implementations of artificial neural networks: Progress update and challenges ahead. Presented at the Oxide-Based Materials and Devices VII, San Francisco, CA, USA, 14-17 February 2016.

16. Cataldo, A.; Biagetti, G.; Mencarelli, D.; Micciulla, F.; Crippa, P.; Turchetti, C.; Pierantoni, L.; Bellucci, S. Modeling and Electrochemical Characterization of Electrodes Based on Epoxy Composite with Functionalized Nanocarbon Fillers at High Concentration. Nanomaterials 2020, 10, 850. [CrossRef]

17. Moon, K.; Lim, S.; Park, J.; Sung, C.; Oh, S.; Woo, J.; Lee, J.; Hwang, H. RRAM-based synapse devices for neuromorphic systems. Faraday Discuss. 2019, 213, 421-451. [CrossRef]

18. Yun, H.J.; Choi, B.J. Effects of moisture and electrode material on AlN-based resistive random access memory. Ceram. Int. 2019, 45, 16311-16316. [CrossRef]

19. Qi, Y.; Shen, Z.; Zhao, C.; Zhao, C.Z. Effect of electrode area on resistive switching behavior in translucent solution-processed $\mathrm{AlO}_{\mathrm{x}}$ based memory device. J. Alloy. Compd. 2020, 822, 153603-153621. [CrossRef]

20. Mahata, C.; Lee, C.; An, Y.; Kim, M.-H.; Bang, S.; Kim, C.S.; Ryu, J.-H.; Kim, S.; Kim, H.; Park, B.-G. Resistive switching and synaptic behaviors of an $\mathrm{HfO}_{2} / \mathrm{Al}_{2} \mathrm{O}_{3}$ stack on ITO for neuromorphic systems. J. Alloy. Compd. 2020, 826, 154434-154460. [CrossRef]

21. Cheng, C.H.; Chin, A.; Hsu, H.H. Forming-Free $\mathrm{SiGeO}_{x} / \mathrm{TiO}_{\mathrm{y}}$ Resistive Random Access Memories Featuring Large Current Distribution Windows. J. Nanosci. Nanotechnol. 2019, 19, 7916-7919. [CrossRef]

22. Ha, S.; Lee, H.; Lee, W.-Y.; Jang, B.; Kwon, H.-J.; Kim, K.; Jang, J. Effect of Annealing Environment on the Performance of Sol-Gel-Processed $\mathrm{ZrO}_{2}$ RRAM. Electronics 2019, 8, 947. [CrossRef]

23. Kim, I.; Siddik, M.; Shin, J.; Biju, K.P.; Jung, S.; Hwang, H. Low temperature solution-processed graphene oxide $/ \operatorname{Pr}_{0.7} \mathrm{Ca}_{0.3} \mathrm{MnO}_{3}$ based resistive-memory device. Appl. Phys. Lett. 2011, 99, 042101-042104. [CrossRef]

24. Hu, Q.; Lee, T.S.; Lee, N.J.; Kang, T.S.; Park, M.R.; Yoon, T.-S.; Lee, H.H.; Kang, C.J. Resistive Switching Characteristics in MnO Nanoparticle Assembly and $\mathrm{Ag}_{2}$ Se Thin Film Devices. J. Nanosci. Nanotechnol. 2017, 17, 7189-7193. [CrossRef]

25. Lin, Y.Y.; Chen, Y.C.; Rettner, C.T.; Raoux, S.; Cheng, H.Y.; Chen, S.H.; Lung, S.L.; Lam, C.; Liu, R. Fast Speed Bipolar Operation of Ge-Sb-Te Based Phase Change Bridge Devices. IEEE Electron. Device Lett. 2008, 8, 462-463.

26. Wu, L.; Guo, J.; Zhong, W.; Zhang, W.; Kang, X.; Chen, W.; Du, Y. Flexible, multilevel, and low-operating-voltage resistive memory based on $\mathrm{MoS}_{2}-\mathrm{rGO}$ hybrid. Appl. Surf. Sci. 2019, 463, 947-952. [CrossRef]

27. Shen, Z.J.; Zhao, C.; Zhao, C.Z.; Mitrovic, I.Z.; Yang, L.; Xu, W.Y.; Lim, E.G.; Luo, T.; Huang, Y.B. Al/GO/Si/Al RRAM with Solution-processed GO dielectric at Low Fabrication Temperature. In Proceedings of the 2019 Joint International EUROSOI Workshop and International Conference on Ultimate Integration on Silicon (EUROSOI-ULIS), Grenoble, France, 1-3 April 2019.

28. Tsai, T.-M.; Lin, C.-C.; Chen, W.-C.; Wu, C.-H.; Yang, C.-C.; Tan, Y.-F.; Wu, P.-Y.; Huang, H.-C.; Zhang, Y.-C.; Sun, L.-C.; et al. Utilizing compliance current level for controllability of resistive switching in nickel oxide thin films for resistive random-access memory. J. Alloy. Compd. 2020, 826, 154126-154151. [CrossRef]

29. Chen, Y. ReRAM: History, Status, and Future. IEEE Trans. Electron. Devices 2020, 67, 1420-1433. [CrossRef]

30. Das, U.; Bhattacharjee, S.; Mahato, B.; Prajapat, M.; Sarkar, P.; Roy, A. Uniform, large-scale growth of WS2 nanodomains via CVD technique for stable non-volatile RRAM application. Mater. Sci. Semicond. Process. 2020, 107, 104837-104842. [CrossRef] 
31. Sun, C.; Lu, S.M.; Jin, F.; Mo, W.Q.; Song, J.L.; Dong, K.F. Multi-factors induced evolution of resistive switching properties for TiN/Gd2O3/Au RRAM devices. J. Alloy. Compd. 2020, 816, 152564-152581. [CrossRef]

32. Roy, S.; Niu, G.; Wang, Q.; Wang, Y.; Zhang, Y.; Wu, H.; Zhai, S.; Shi, P.; Song, S.; Song, Z.; et al. Toward a Reliable Synaptic Simulation Using Al-Doped HfO2 RRAM. ACS Appl. Mater. Interfaces 2020, 12, 10648-10656. [CrossRef]

33. Cui, X.; Li, X.; Zhang, M.; Cui, X. Design of the RRAM-Based Polymorphic Look-Up Table Scheme. IEEE J. Electron. Devices Soc. 2019, 7, 949-953. [CrossRef]

34. Chuang, K.-C.; Chu, C.-Y.; Zhang, H.-X.; Luo, J.-D.; Li, W.-S.; Li, Y.-S.; Cheng, H.-C. Impact of the Stacking Order of HfOx and AlOx Dielectric Films on RRAM Switching Mechanisms to Behave Digital Resistive Switching and Synaptic Characteristics. IEEE J. Electron. Devices Soc. 2019, 7, 589-595. [CrossRef]

35. Mao, J.-Y.; Zhou, L.; Ren, Y.; Yang, J.-Q.; Chang, C.-L.; Lin, H.-C.; Chou, H.-H.; Zhang, S.-R.; Zhou, Y.; Han, S.-T. A bio-inspired electronic synapse using solution processable organic small molecule. J. Mater. Chem. C 2019, 7, 1491-1501. [CrossRef]

36. Lee, D.K.; Kim, M.-H.; Kim, T.-H.; Bang, S.; Choi, Y.-J.; Kim, S.; Cho, S.; Park, B.-G. Synaptic behaviors of HfO2 ReRAM by pulse frequency modulation. Solid State Electron. 2019, 154, 31-35. [CrossRef]

37. Yang, S.; Hao, X.; Deng, B.; Wei, X.; Li, H.; Wang, J. A survey of brain-inspired artificial intelligence and its engineering. Life Res. 2018, 1, 23-29.

38. Wang, H.; Yan, X. Overview of Resistive Random Access Memory (RRAM): Materials, Filament Mechanisms, Performance Optimization, and Prospects. Phys. Status Solidi RRL Rapid Res. Lett. 2019, 13, 1900073-1900084. [CrossRef]

39. Zhang, X.; Xu, L.; Zhang, H.; Liu, J.; Tan, D.; Chen, L.; Ma, Z.; Li, W. Effect of Joule Heating on Resistive Switching Characteristic in AlOx Cells Made by Thermal Oxidation Formation. Nanoscale Res. Lett. 2020, 19, 3229-1-3229-19. [CrossRef] [PubMed]

40. Tsuruoka, T.; Hasegawa, T.; Terabe, K.; Aono, M. Conductance quantization and synaptic behavior in a Ta2O5-based atomic switch. Nanotechnology 2012, 23, 435705-435711. [CrossRef]

41. Sun, P.; Li, L.; Lu, N.; Li, Y.; Wang, M.; Xie, H.; Liu, S.; Liu, M. Physical model of dynamic Joule heating effect for reset process in conductive-bridge random access memory. J. Comput. Electron. 2014, 13, 432-438. [CrossRef]

42. Zhang, T.; Ou, X.; Zhang, W.; Yin, J.; Xia, Y.; Liu, Z. High-k-rare-earth-oxide Eu2O3 films for transparent resistive random access memory (RRAM) devices. J. Phys. D Appl. Phys. 2014, 47, 065302-065308. [CrossRef]

43. Chen, K.J.; Liu, J.; Wang, Y.; Yang, H.; Ma, Z.; Huang, X. VCM Conductive defect states based filament in MOM structure RRAM. IEEE Electron. Device Lett. 2018, 9, 1-4. [CrossRef]

44. Kwon, D.H.; Kim, K.M.; Jang, J.H.; Jeon, J.M.; Lee, M.H.; Kim, G.H.; Li, X.S.; Park, G.S.; Lee, B.; Han, S.; et al. Atomic structure of conducting nanofilaments in TiO2 resistive switching memory. Nat. Nanotechnol 2010, 5, 148-153. [CrossRef] [PubMed]

45. Xue, W.; Liu, G.; Zhong, Z.; Dai, Y.; Shang, J.; Liu, Y.; Yang, H.; Yi, X.; Tan, H.; Pan, L.; et al. A 1D Vanadium Dioxide Nanochannel Constructed via Electric-Field-Induced Ion Transport and its Superior Metal-Insulator Transition. Adv. Mater. 2017, 29, 1702162(1)-1702162(9). [CrossRef] [PubMed]

46. Lee, S.; Sohn, J.; Jiang, Z.; Chen, H.Y.; Philip Wong, H.S. Metal oxide-resistive memory using graphene-edge electrodes. Nat. Commun. 2015, 6, 8407-8413. [CrossRef] [PubMed]

47. Munjal, S.; Khare, N. Valence Change Bipolar Resistive Switching Accompanied With Magnetization Switching in CoFe2O4 Thin Film. Sci. Rep. 2017, 7, 12427-12436. [CrossRef] [PubMed]

48. Ye, C.; Wu, J.; He, G.; Zhang, J.; Deng, T.; He, P.; Wang, H. Physical Mechanism and Performance Factors of Metal Oxide Based Resistive Switching Memory: A Review. J. Mater. Sci. Technol. 2016, 32, 1-11. [CrossRef]

49. Yu, D.; Liu, L.F.; Chen, B.; Zhang, F.F.; Gao, B.; Fu, Y.H.; Liu, X.Y.; Kang, J.F.; Zhang, X. Multilevel resistive switching characteristics in Ag/SiO2/Pt RRAM devices. IEEE Electron. Device Lett. 2011, 11, 1-4.

50. Tsuruoka, T.; Terabe, K.; Hasegawa, T.; Aono, M. Forming and switching mechanisms of a cation-migrationbased oxide resistive memory. Nanotechnology 2010, 21, 425205-425213. [CrossRef]

51. Guo, T.; Sun, B.; Zhou, Y.; Zhao, H.; Lei, M.; Zhao, Y. Overwhelming coexistence of negative differential resistance effect and RRAM. Phys. Chem Chem Phys. 2018, 20, 20635-20640. [CrossRef]

52. Long, S.B.; Liu, Q.; Lv, H.B.; Li, Y.T.; Wang, Y.; Zhang, S.; Lian, W.-T.; Liu, M. Resistive switching mechanism of $\mathrm{Cu}$ doped $\mathrm{ZrO} 2$-based RRAM. IEEE Electron. Device Lett. 2010, 5, 1-4. 
53. Long, S.; Liu, Q.; Lv, H.; Li, Y.; Wang, Y.; Zhang, S.; Lian, W.; Zhang, K.; Wang, M.; Xie, H.; et al. Resistive switching mechanism of Ag/ZrO2:Cu/Pt memory cell. Appl. Phys. A 2011, 102, 915-919. [CrossRef]

54. Yuan, F.; Shen, S.; Zhang, Z.; Pan, L.; Xu, J. Interface-induced two-step RESET for filament-based multi-level resistive memory. Superlattices Microstruct. 2016, 91, 90-97. [CrossRef]

55. Gao, S.; Zeng, F.; Chen, C.; Tang, G.; Lin, Y.; Zheng, Z.; Song, C.; Pan, F. Conductance quantization in a Ag filament-based polymer resistive memory. Nanotechnology 2013, 24, 335201-335208. [CrossRef] [PubMed]

56. Ren, Y.; Milo, V.; Wang, Z.; Xu, H.; Ielmini, D.; Zhao, X.; Liu, Y. Analytical Modeling of Organic-Inorganic $\mathrm{CH} 3 \mathrm{NH} 3 \mathrm{PbI} 3$ Perovskite Resistive Switching and its Application for Neuromorphic Recognition. Adv. Theory Simul. 2018, 1, 1700035-1700042. [CrossRef]

57. Larentis, S.; Cagli, C.; Nardi, F.; Ielmini, D. Filament diffusion model for simulating reset and retention processes in RRAM. Microelectron. Eng. 2011, 88, 1119-1123. [CrossRef]

58. Ambrogio, S.; Balatti, S.; Gilmer, D.C.; Ielmini, D. Analytical Modeling of Oxide-Based Bipolar Resistive Memories and Complementary Resistive Switches. IEEE Trans. Electron. Devices 2014, 61, 2378-2386. [CrossRef]

59. Ielmini, D. Modeling the Universal Set/Reset Characteristics of Bipolar RRAM by Field- and Temperature-Driven Filament Growth. IEEE Trans. Electron. Devices 2011, 58, 4309-4317. [CrossRef]

60. Zhou, K.-J.; Chang, T.-C.; Lin, C.-Y.; Chen, C.-K.; Tseng, Y.-T.; Zheng, H.-X.; Chen, H.-C.; Sun, L.-C.; Lien, C.-Y.; Tan, Y.-F.; et al. Abnormal High Resistive State Current Mechanism Transformation in Ti/HfO2/TiN Resistive Random Access Memory. IEEE Electron. Device Lett. 2020, 41, 224-227. [CrossRef]

61. Sheridan, P.; Kim, K.H.; Gaba, S.; Chang, T.; Chen, L.; Lu, W. Device and SPICE modeling of RRAM devices. Nanoscale 2011, 3, 3833-3840. [CrossRef]

62. Shen, Z.; Zhao, C.; Qi, Y.; Mitrovic, I.Z.; Yang, L.; Wen, J.; Huang, Y.; Li, P.; Zhao, C. Memristive Non-Volatile Memory Based on Graphene Materials. Micromachines 2020, 11, 341. [CrossRef]

63. Xiao, W.; Song, W.; Feng, Y.P.; Gao, D.; Zhu, Y.; Ding, J. Electrode-controlled confinement of conductive filaments in a nanocolumn embedded symmetric-asymmetric RRAM structure. J. Mater. Chem. C 2020, 8 , 1577-1582. [CrossRef]

64. Mao, S.; Zhou, G.; Sun, B.; Elshekh, H.; Zhang, X.; Xia, Y.; Yang, F.; Zhao, Y. Mechanism analysis of switching direction transformation in an Er2O3 based RRAM device. Curr. Appl. Phys. 2019, 19, 1421-1426. [CrossRef]

65. Wang, H.; Zhu, B.; Ma, X.; Hao, Y.; Chen, X. Physically Transient Resistive Switching Memory Based on Silk Protein. Small 2016, 12, 2715-2719. [CrossRef] [PubMed]

66. Wang, H.; Zhu, B.; Wang, H.; Ma, X.; Hao, Y.; Chen, X. Ultra-Lightweight Resistive Switching Memory Devices Based on Silk Fibroin. Small 2016, 12, 3360-3365. [CrossRef] [PubMed]

67. Chen, Y.C.; Yu, H.C.; Huang, C.Y.; Chung, W.L.; Wu, S.L.; Su, Y.K. Nonvolatile Bio-Memristor Fabricated with Egg Albumen Film. Sci. Rep. 2014, 5, 1002201-1002212. [CrossRef] [PubMed]

68. Méhes, G.; Vagin, M.; Mulla, M.Y.; Granberg, H.; Che, C.; Beni, V.; Crispin, X.; Berggren, M.; Stavrinidou, E.; Simon, D.T. Simon, Solar Heat-Enhanced Energy Conversion in Devices Based on Photosynthetic Membranes and PEDOT PSS-Nanocellulose Electrodes. Adcanced Sustain. Syst. 2019, 19, 1900100.

69. Poskela, A.; Miettunen, K.; Borghei, M.; Vapaavuori, J.; Greca, L.G.; Lehtonen, J.; Solin, K.; Ago, M.; Lund, P.D.; Rojas, O.J. Nanocellulose and Nanochitin Cryogels Improve the Efficiency of Dye Solar Cells. ACS Sustain. Chem. Eng. 2019, 7, 10257-10265.

70. Jin, H.; Li, J.; Iocozzia, J.; Zeng, X.; Wei, P.C.; Yang, C.; Li, N.; Liu, Z.; He, J.H.; Zhu, T.; et al. Hybrid Organic-Inorganic Thermoelectric Materials and Devices. Angew. Chem. Int. Ed. Engl. 2019, 58, 15206-15226. [CrossRef]

71. Yarimaga, O.; Lee, S.; Ham, D.-Y.; Choi, J.-M.; Kwon, S.G.; Im, M.; Kim, S.; Kim, J.-M.; Choi, Y.-K. Thermofluorescent Conjugated Polymer Sensors for Nano- and Microscale Temperature Monitoring. Macromol. Chem. Phys. 2011, 212,1211-1220.

72. Ling, Q.D.; Lim, S.L.; Song, Y.; Zhu, C.X.; Chan DS, H.; Kang, E.T.; Neoh, K.G. Nonvolatile Polymer Memory Device Based on Bistable Electrical Switching in a Thin Film of Poly(N-vinylcarbazole) with Covalently Bonded C60. Langmuir 2007, 23, 312-319. [CrossRef]

73. Zhu, Y.; Cheng, P.; Shi, J.; Wang, H.; Liu, Y.; Xiong, R.; Ma, H.; Ma, H. Bromine Vacancy Redistribution and Metallic-Ion-Migration-Induced Air-Stable Resistive Switching Behavior in All-Inorganic Perovskite $\mathrm{CsPbBr}_{3}$ Film-Based Memory Device. Adv. Electron. Mater. 2019, 6, 1900754-1900761. [CrossRef]

74. Khurana, G.; Misra, P.; Katiyar, R.S. Forming free resistive switching in graphene oxide thin film for thermally stable nonvolatile memory applications. J. Appl. Phys. 2013, 114, 124508-124514. [CrossRef] 
75. Gan, K.-J.; Chang, W.-C.; Liu, P.-T.; Sze, S.M. Investigation of resistive switching in copper/InGaZnO/ $\mathrm{Al}_{2} \mathrm{O}_{3}$-based memristor. Appl. Phys. Lett. 2019, 115, 143501-143506. [CrossRef]

76. Lee, B.R.; Park, J.H.; Kim, T.G. Micro-light-emitting diode with n-GaN/NiO/Au-based resistive-switching electrode for compact driving circuitry. J. Alloy. Compd. 2020, 823, 153762-153767. [CrossRef]

77. Sung, C.; Lim, S.; Hwang, H. Experimental Determination of the Tunable Threshold Voltage Characteristics in a $\mathrm{Ag}_{\mathrm{x}} \mathrm{Te}_{1-\mathrm{x}} / \mathrm{Al}_{2} \mathrm{O}_{3} / \mathrm{TiO}_{2}$-based Hybrid Memory Device. IEEE Electron Device Lett. 2020, 41, 713-716.

78. Kim, J.; Cho, S.; Kim, T.; Pak, J.J. Mimicking Synaptic Behaviors with Cross-Point Structured TiO $\mathrm{T}_{\mathrm{TiO}}-\mathrm{Based}$ Filamentary RRAM for Neuromorphic Applications. J. Electr. Eng. Technol. 2019, 14, 869-875. [CrossRef]

79. Wang, Q.; Niu, G.; Roy, S.; Wang, Y.; Zhang, Y.; Wu, H.; Zhai, S.; Bai, W.; Shi, P.; Song, S.; et al. Interface-engineered reliable $\mathrm{HfO}_{2}$-based RRAM for synaptic simulation. J. Mater. Chem. C 2019, 7, 12682-12687. [CrossRef]

80. Cai, L.; Chen, W.; Zhao, Y.; Liu, X.; Kang, J.; Zhang, X.; Huang, P. Insight into Effects of Oxygen Reservoir Layer and Operation Schemes on Data Retention of $\mathrm{HfO}_{2}$-Based RRAM. IEEE Trans. Electron. Devices 2019, 66, 3822-3827. [CrossRef]

81. Gupta, C.P.; Jain, P.K.; Chand, U.; Sharma, S.K.; Birla, S.; Sancheti, S. Effect of Top Electrode Materials on Switching Characteristics and Endurance Properties of Zinc Oxide Based RRAM Device. J. Nano Electron. Phys. 2020, 12, 01007-1-01007-6. [CrossRef]

82. Kumar, D.; Chand, U.; Wen Siang, L.; Tseng, T.-Y. High-Performance TiN/ $/ \mathrm{Al}_{2} \mathrm{O}_{3} / \mathrm{ZnO} / \mathrm{Al}_{2} \mathrm{O}_{3} / \mathrm{TiN}$ Flexible RRAM Device With High Bending Condition. IEEE Trans. Electron. Devices 2020, 67, 493-498. [CrossRef]

83. Wu, P.-Y.; Zheng, H.-X.; Shih, C.-C.; Chang, T.-C.; Chen, W.-J.; Yang, C.-C.; Chen, W.-C.; Tai, M.-C.; Tan, Y.-F.; Huang, H.-C.; et al. Improvement of Resistive Switching Characteristics in Zinc Oxide-Based Resistive Random Access Memory by Ammoniation Annealing. IEEE Electron. Device Lett. 2020, 41, 357-360. [CrossRef]

84. Hussain, F.; Imran, M.; Khalil, R.M.A.; Sattar, M.A.; Niaz, N.A.; Rana, A.M.; Ismail, M.; Khera, E.A.; Rasheed, U.; Mumtaz, F; et al. A first-principles study of $\mathrm{Cu}$ and $\mathrm{Al}$ doping in $\mathrm{ZrO}_{2}$ for $\mathrm{RRAM}$ device applications. Vacuum 2019, 168, 108842-108847. [CrossRef]

85. Wei, X.; Huang, H.; Ye, C.; Wei, W.; Zhou, H.; Chen, Y.; Zhang, R.; Zhang, L.; Xia, Q. Exploring the role of nitrogen incorporation in $\mathrm{ZrO}_{2}$ resistive switching film for enhancing the device performance. J. Alloy. Compd. 2019, 775, 1301-1306. [CrossRef]

86. Liu, K.-C.; Tzeng, W.-H.; Chang, K.-M.; Huang, J.-J.; Lee, Y.-J.; Yeh, P.-H.; Chen, P.-S.; Lee, H.-Y.; Chen, F.; Tsai, M.-J. Investigation of the effect of different oxygen partial pressure to $\mathrm{LaAlO}_{3}$ thin film properties and resistive switching characteristics. Thin Solid Film. 2011, 520, 1246-1250. [CrossRef]

87. Kim, C.H.; Ahn, Y.; Son, J.Y. SrTiO 3 -Based Resistive Switching Memory Device with Graphene Nanoribbon Electrodes. Rapid Commun. 2016, 99, 9-11. [CrossRef]

88. Chen, S.-W.; Wu, J.-M. Unipolar resistive switching behavior of $\mathrm{BiFeO}_{3}$ thin films prepared by chemical solution deposition. Thin Solid Films 2010, 519, 499-504. [CrossRef]

89. Chen, H.Y.; Tian, H.; Gao, B.; Yu, S.; Liang, J.; Kang, J.; Zhang, Y.; Ren, T.-L.; Wong, H.S.P. Electrode oxide interface engineering by inserting single-layer graphene Application for $\mathrm{HfO}_{\mathrm{x}}$-based resistive random access memory. IEEE Electron. Device Lett. 2012, 11, 489-492.

90. Chen, Z.; Zhang, Y.; Yu, Y.; Che, Y.; Jin, L.; Li, Y.; Li, Q.; Li, T.; Dai, H.; Yao, J. Write once read many times resistance switching memory based on all-inorganic perovskite $\mathrm{CsPbBr}_{3}$ quantum dot. Opt. Mater. 2019, 90, 123-126. [CrossRef]

91. Hickmott, T.W. Low-Frequency Negative Resistance in Thin Anodic Oxide Films. J. Appl. Phys. 1962, 33, 2669-2682. [CrossRef]

92. Samanta, S.; Han, K.; Das, S.; Gong, X. Improvement in Threshold Switching Performance Using $\mathrm{Al}_{2} \mathrm{O}_{3}$ Interfacial Layer in $\mathrm{Ag} / \mathrm{Al}_{2} \mathrm{O}_{3} / \mathrm{SiO}_{x} / \mathrm{W}$ Cross-Point Platform. IEEE Electron. Device Lett. 2020, 41, 924-927. [CrossRef]

93. Banerjee, W.; Rahaman, S.Z.; Maikap, S. Excellent Uniformity and Multilevel Operation in Formation-Free Low Power Resistive Switching Memory Using $\mathrm{IrO}_{\mathrm{x}} / \mathrm{AlO}_{\mathrm{x}} / \mathrm{W}$ Cross-Point. Jpn. J. Appl. Phys. 2012, 51, 04dd10-04dd16. [CrossRef]

94. Knorr, N.; Bamedi, A.; Karipidou, Z.; Wirtz, R.; Sarpasan, M.; Rosselli, S.; Nelles, G. Evidence of electrochemical resistive switching in the hydrated alumina layers of $\mathrm{Cu} / \mathrm{CuTCNQ} /($ native $\mathrm{AlOx}) / \mathrm{Al}$ junctions. J. Appl. Phys. 2013, 114, 124510-124518. [CrossRef] 
95. Sleiman, A.; Sayers, P.W.; Mabrook, M.F. Mechanism of resistive switching in $\mathrm{Cu} / \mathrm{AlO}$ x/W nonvolatile memory structures. J. Appl. Phys. 2013, 113, 164506-164511. [CrossRef]

96. Anwar, F.; Nogan, J.; Zarkesh-Ha, P.; Osinski, M. Multilevel resistance in Ti/Pt/AlO $/ \mathrm{HfO}_{\mathrm{y}} / \mathrm{Ti} / \mathrm{Pt} / \mathrm{Ag}$ resistive switching devices. In Proceedings of the 2015 IEEE Nanotechnology Materials and Devices Conference (NMDC), Anchorage, AK, USA, 13-16 September 2015.

97. Varun, I.; Bharti, D.; Raghuwanshi, V.; Tiwari, S.P. Multi-temperature deposition scheme for improved resistive switching behavior of Ti/AlO $/$ /Ti MIM structure. Solid State Ion. 2017, 309, 86-91. [CrossRef]

98. Kurnia, F.; Liu, C.; Raveendra, N.; Jung, C.U.; Vasudevan, R.K.; Valanoor, N. Self-Assembled NiO Nanocrystal Arrays as Memristive Elements. Adv. Electron. Mater. 2020, 6, 1901153-1901160. [CrossRef]

99. Yoshida, C.; Kinoshita, K.; Yamasaki, T.; Sugiyama, Y. Direct observation of oxygen movement during resistance switching in NiO/Pt film. Appl. Phys. Lett. 2008, 93, 042106-042109. [CrossRef]

100. Russo, U.; Ielmini, D.; Cagli, C.; Lacaita, A.L. Filament Conduction and Reset Mechanism in NiO-Based Resistive-Switching Memory (RRAM) Devices. IEEE Trans. Electron. Devices 2009, 56, 186-192. [CrossRef]

101. Cagli, C.; Nardi, F.; Ielmini, D. Modeling of Set/Reset Operations in NiO-Based Resistive-Switching Memory Devices. IEEE Trans. Electron. Devices 2009, 56, 1712-1720. [CrossRef]

102. Ielmini, D.; Nardi, F.; Cagli, C.; Lacaita, A.L. Size-Dependent Retention Time in NiO-Based Resistive-Switching Memories. IEEE Electron. Device Lett. 2010, 31, 353-355. [CrossRef]

103. Sullaphen, J.; Bogle, K.; Cheng, X.; Gregg, J.M.; Valanoor, N. Interface mediated resistive switching in epitaxial NiO nanostructures. Appl. Phys. Lett. 2012, 100, 203115-203120. [CrossRef]

104. Sakellaropoulos, D.; Bousoulas, P.; Nikas, G.; Arvanitis, C.; Bagakis, E.; Tsoukalas, D. Enhancing the synaptic properties of low-power and forming-free $\mathrm{HfO}_{\mathrm{x}} / \mathrm{TaO}_{\mathrm{y}} / \mathrm{HfO}_{\mathrm{x}}$ resistive switching devices. Microelectron. Eng. 2020, 229, 111358-111364. [CrossRef]

105. Nauenheim, C.; Kuegeler, C.; Ruediger, A.; Waser, R. Investigation of the electroforming process in resistively switching $\mathrm{TiO}_{2}$ nanocrosspoint junctions. Appl. Phys. Lett. 2010, 96, 122902-122905. [CrossRef]

106. Hermes, C.; Bruchhaus, R.; Waser, R. Forming-Free $\mathrm{TiO}_{2}$-Based Resistive Switching Devices on CMOS-Compatible W-Plugs. IEEE Electron. Device Lett. 2011, 32, 1588-1590. [CrossRef]

107. Salaoru, I.; Prodromakis, T.; Khiat, A.; Toumazou, C. Resistive switching of oxygen enhanced $\mathrm{TiO}_{2}$ thin-film devices. Appl. Phys. Lett. 2013, 102, 013506-013510. [CrossRef]

108. Otsuka, S.; Hamada, Y.; Shimizu, T.; Shingubara, S. Ferromagnetic nano-conductive filament formed in $\mathrm{Ni} / \mathrm{TiO}_{2} / \mathrm{Pt}$ resistive-switching memory. Appl. Phys. A 2014, 118, 613-619. [CrossRef]

109. Vishwanath, S.K.; Kim, J. Resistive switching characteristics of all-solution-based Ag/TiO $/ \mathrm{Mo}$-doped $\mathrm{In}_{2} \mathrm{O}_{3}$ devices for non-volatile memory applications. J. Mater. Chem. C 2016, 4, 10967-10972. [CrossRef]

110. Sharath, S.U.; Bertaud, T.; Kurian, J.; Hildebrandt, E.; Walczyk, C.; Calka, P.; Zaumseil, P.; Sowinska, M.; Walczyk, D.; Gloskovskii, A.; et al. Towards forming-free resistive switching in oxygen engineered $\mathrm{HfO}_{2-\mathrm{x}}$. Appl. Phys. Lett. 2014, 104, 063502-063507. [CrossRef]

111. Clima, S.; Chen, Y.Y.; Degraeve, R.; Mees, M.; Sankaran, K.; Govoreanu, B.; Jurczak, M.; De Gendt, S.; Pourtois, G. First-principles simulation of oxygen diffusion in $\mathrm{HfO}_{\mathrm{x}}$ : Role in the resistive switching mechanism. Appl. Phys. Lett. 2012, 100, 133102-133106. [CrossRef]

112. Lanza, M.; Zhang, K.; Porti, M.; Nafría, M.; Shen, Z.Y.; Liu, L.F.; Kang, J.F.; Gilmer, D.; Bersuker, G. Grain boundaries as preferential sites for resistive switching in the $\mathrm{HfO}_{2}$ resistive random access memory structures. Appl. Phys. Lett. 2012, 100, 123508. [CrossRef]

113. De Stefano, F.; Houssa, M.; Kittl, J.A.; Jurczak, M.; Afanas'ev, V.V.; Stesmans, A. Semiconducting-like filament formation in $\mathrm{TiN} / \mathrm{HfO}_{2} / \mathrm{TiN}$ resistive switching random access memories. Appl. Phys. Lett. 2012, 100, 142102-142105. [CrossRef]

114. Ambrogio, S.; Balatti, S.; Cubeta, A.; Calderoni, A.; Ramaswamy, N.; Ielmini, D. Statistical Fluctuations in $\mathrm{HfO}_{\mathrm{x}}$ Resistive-Switching Memory: Part I - Set/Reset Variability. IEEE Trans. Electron. Devices 2014, 61, 2912-2919. [CrossRef]

115. Abbas, Y.; Han, I.S.; Sokolov, A.S.; Jeon, Y.-R.; Choi, C. Rapid thermal annealing on the atomic layer-deposited zirconia thin film to enhance resistive switching characteristics. J. Mater. Sci. Mater. Electron. 2019, 31, 903-909. [CrossRef]

116. Verbakel, F.; Meskers, S.C.; de Leeuw, D.M.; Janssen, R.A. Resistive Switching in Organic Memories with a Spin-Coated Metal Oxide Nanoparticle Layer. J. Phys. Chem. C Lett. 2008, 112, 5254-5257. [CrossRef] 
117. Awais, M.N.; Muhammad, N.M.; Navaneethan, D.; Kim, H.C.; Jo, J.; Choi, K.H. Fabrication of ZrO $\mathrm{Zr}_{2}$ layer through electrohydrodynamic atomization for the printed resistive switch (memristor). Microelectron. Eng. 2013, 103, 167-172. [CrossRef]

118. Kärkkänen, I.; Shkabko, A.; Heikkilä, M.; Niinistö, J.; Ritala, M.; Leskelä, M.; Hoffmann-Eifert, S.; Waser, R. Study of atomic layer deposited $\mathrm{ZrO}_{2}$ and $\mathrm{ZrO}_{2} / \mathrm{TiO}_{2}$ films for resistive switching application. Phys. Status Solidi (a) 2014, 211, 301-309. [CrossRef]

119. Ismail, M.; Huang, C.Y.; Panda, D.; Hung, C.J.; Tsai, T.L.; Jieng, J.H.; Lin, C.-A.; Chand, U.; Rana, A.M.; Ahmed, E.; et al. Muhammad Younus Nadeem and Tseung-Yuen Tseng, Forming-free bipolar resistive switching in nonstoichiometric ceria films. Nanoscale Res. Lett. 2014, 9, 1-8. [CrossRef] [PubMed]

120. Parreira, P.; Paterson, G.W.; McVitie, S.; MacLaren, D.A. Stability, bistability and instability of amorphous $\mathrm{ZrO}_{2}$ resistive memory devices. J. Phys. D Appl. Phys. 2016, 49, 095111-095117. [CrossRef]

121. Bailey, T.J.; Jha, R. Understanding Synaptic Mechanisms in $\mathrm{SrTiO}_{3}$ RRAM Devices. IEEE Trans. Electron. Devices 2018, 65, 3514-3520. [CrossRef]

122. Tsubouchi, K.; Ohkubo, I.; Kumigashira, H.; Oshima, M.; Matsumoto, Y.; Itaka, K.; Ohnishi, T.; Lippmaa, M.; Koinuma, H. High-Throughput Characterization of Metal Electrode Performance for Electric-Field-Induced Resistance Switching in Metal $\mathrm{Pr}_{0.7} \mathrm{Ca}_{0.3} \mathrm{MnO}_{3}$ Metal Structure. Adv. Mater. 2007, 19, 1711-1713. [CrossRef]

123. Hirose, Y.; Hirose, H. Polarity-dependent memory switching and behavior of Ag dendrite in Ag-photodoped amorphous $\mathrm{As}_{2} \mathrm{~S}_{3}$ films. J. Appl. Phys. 1976, 47, 2767-2772. [CrossRef]

124. Imanishi, Y.; Kida, S.; Nakaoka, T. Direct observation of Ag filament growth and unconventional SET-RESET operation in GeTe amorphous films. AIP Adv. 2016, 6, 075003-1-075003-9. [CrossRef]

125. Bai, Y.; Wu, H.; Wang, K.; Wu, R.; Song, L.; Li, T.; Wang, J.; Yu, Z.; Qian, H. Stacked 3D RRAM Array with Graphene/CNT as Edge Electrodes. Sci Rep. 2015, 5, 13785-13793. [CrossRef] [PubMed]

126. Jeon, H.; Park, J.; Jang, W.; Kim, H.; Ahn, S.; Jeon, K.-J.; Seo, H.; Jeon, H. Detection of oxygen ion drift in $\mathrm{Pt} / \mathrm{Al}_{2} \mathrm{O}_{3} / \mathrm{TiO}_{2} / \mathrm{Pt} \mathrm{RRAM}$ using interface-free single-layer graphene electrodes. Carbon 2014, 75, 209-216. [CrossRef]

127. Xia, Y.; Sun, B.; Wang, H.; Zhou, G.; Kan, X.; Zhang, Y.; Zhao, Y. Metal ion formed conductive filaments by redox process induced nonvolatile resistive switching memories in $\mathrm{MoS}_{2}$ film. Appl. Surf. Sci. 2017, 426, 812-816. [CrossRef]

128. Kadhim, M.S.; Yang, F.; Sun, B.; Hou, W.; Peng, H.; Hou, Y.; Jia, Y.; Yuan, L.; Yu, Y.; Zhao, Y. Existence of Resistive Switching Memory and Negative Differential Resistance State in Self-Colored $\mathrm{MoS}_{2} / \mathrm{ZnO}$ Heterojunction Devices. ACS Appl. Electron. Mater. 2019, 1, 318-324. [CrossRef]

129. Chen, C.; Gao, S.; Zeng, F.; Tang, G.S.; Li, S.Z.; Song, C.; Fu, H.D.; Pan, F. Migration of interfacial oxygen ions modulated resistive switching in oxide-based memory devices. J. Appl. Phys. 2013, 114, 014502-014509. [CrossRef]

130. Jana, D.; Dutta, M.; Samanta, S.; Maikap, S. Subhranu Samanta and Siddheswar Maikap, RRAM characteristics using a new $\mathrm{Cr} \mathrm{GdO}_{x}$ TiN structure. Nanoscale Res. Lett. 2014, 9, 680-1-680-9. [CrossRef]

131. Hongbin, Z.; Hailing, T.; Feng, W.; Jun, D. Highly Transparent Dysprosium Oxide-Based RRAM with Multilayer Graphene Electrode for Low-Power Nonvolatile Memory Application. IEEE Trans. Electron. Devices 2014, 61, 1388-1393. [CrossRef]

132. Chen, S.-C.; Chang, T.-C.; Chen, S.-Y.; Chen, C.-W.; Chen, S.-C.; Sze, S.M.; Tsai, M.-J.; Kao, M.-J.; Yeh, F.-S. Bipolar resistive switching of chromium oxide for resistive random access memory. Solid State Electron. 2011, 62, 40-43. [CrossRef]

133. Huang, Y.; Huang, R.; Pan, Y.; Zhang, L.; Cai, Y.; Yang, G.; Wang, Y. A New Dynamic Selector Based on the Bipolar RRAM for the Crossbar Array Application. IEEE Trans. Electron. Devices 2012, 59, 2277-2280. [CrossRef]

134. Park, K.; Lee, J.S. Controlled synthesis of $\mathrm{Ni} / \mathrm{CuO}_{\mathrm{x}} / \mathrm{Ni}$ nanowires by electrochemical deposition with self-compliance bipolar resistive switching. Sci. Rep. 2016, 6, 23069-23074. [CrossRef] [PubMed]

135. Seo, S.; Lim, J.; Lee, S.; Alimkhanuly, B.; Kadyrov, A.; Jeon, D.; Lee, S. Graphene-Edge Electrode on a Cu-Based Chalcogenide Selector for 3D Vertical Memristor Cells. ACS Appl Mater. Interfaces 2019, 11, 43466-43472. [CrossRef] [PubMed]

136. Chang, T.; Jo, S.H.; Lu, W. Short-Term Memory to Long-Term memory transition in a nanoscale memristor. ACS Nano 2011, 5, 7669-7676. [CrossRef] [PubMed] 
137. Ambrogio, S.; Balatti, S.; Milo, V.; Carboni, R.; Wang, Z.-Q.; Calderoni, A.; Ramaswamy, N.; Ielmini, D. Neuromorphic Learning and Recognition With One-Transistor-One-Resistor Synapses and Bistable Metal Oxide RRAM. IEEE Trans.Electron. Devices 2016, 63, 1508-1515. [CrossRef]

138. Wang, S.; Zhang, D.W.; Zhou, P. Two-dimensional materials for synaptic electronics and neuromorphic systems. Sci. Bull. 2019, 64, 1056-1066. [CrossRef]

139. Shi, Y.; Liang, X.; Yuan, B.; Chen, V.; Li, H.; Hui, F.; Yu, Z.; Yuan, F.; Pop, E.; Wong, H.S.P.; et al. Electronic synapses made of layered two-dimensional materials. Nat. Electron. 2018, 1, 458-465. [CrossRef]

140. Wang, Z.; Zeng, T.; Ren, Y.; Lin, Y.; Xu, H.; Zhao, X.; Liu, Y.; Ielmini, D. Toward a generalized Bienenstock-Cooper-Munro rule for spatiotemporal learning via triplet-STDP in memristive devices. Nat. Commun. 2020, 11, 1509-1510. [CrossRef]

141. Ielmini, D. Brain-inspired computing with resistive switching memory (RRAM): Devices, synapses and neural networks. Microelectron. Eng. 2018, 190, 44-53. [CrossRef]

142. Kim, B.; Choi, H.-S.; Kim, Y. A Study of Conductance Update Method for Ni/SiN $\mathrm{S}_{\mathrm{x}} / \mathrm{Si}$ Analog Synaptic Device. Solid State Electron. 2020, 171, 107772-107799. [CrossRef]

143. Serb, A.; Corna, A.; George, R.; Khiat, A.; Rocchi, F.; Reato, M.; Maschietto, M.; Mayr, C.; Indiveri, G.; Vassanelli, S.; et al. Memristive synapses connect brain and silicon spiking neurons. Sci. Rep. 2020, 10, 2590-2597. [CrossRef]

144. Liu, B.; Tai, H.H.; Liang, H.; Zheng, E.-Y.; Sahoo, M.; Hsu, C.-H.; Chen, T.C.; Huang, C.-A.; Wang, J.-C.; Hou, T.-H.; et al. Dimensional Anisotropic Graphene with High Mobility and High On-Off Ratio in three-terminal RRAM device. Mater. Chem. Front. 2020, 10, 1-17.

145. Park, Y.; Lee, J.S. Artificial Synapses with Short- and Long-Term Memory for Spiking Neural Networks Based on Renewable Materials. ACS Nano 2017, 11, 8962-8969. [CrossRef] [PubMed]

146. Kim, H.; Bae, J.-H.; Lim, S.; Lee, S.-T.; Seo, Y.-T.; Kwon, D.; Park, B.-G.; Lee, J.-H. Efficient precise weight tuning protocol considering variation of the synaptic devices and target accuracy. Neurocomputing 2020, 378, 189-196. [CrossRef]

147. Halter, M.; Begon-Lours, L.; Bragaglia, V.; Sousa, M.; Offrein, B.J.; Abel, S.; Luisier, M.; Fompeyrine, J. Back-End, CMOS-Compatible Ferroelectric Field-Effect Transistor for Synaptic Weights. ACS Appl. Mater. Interfaces 2020, 12, 17725-17732. [CrossRef] [PubMed]

148. Song, S.; Miller, K.D.; Abbott, L.F. Competitive Hebbian learning through spike-timing-dependent synaptic plasticity. Nat. Neurosci. 2000, 3, 919-926. [CrossRef] [PubMed]

149. Patil, V.L.; Patil, A.A.; Patil, S.V.; Khairnar, N.A.; Tarwal, N.L.; Vanalakar, S.A.; Bulakhe, R.N.; In, I.; Patil, P.S.; Dongale, T.D. Bipolar resistive switching, synaptic plasticity and non-volatile memory effects in the solution-processed zinc oxide thin film. Mater. Sci. Semicond. Process. 2020, 106, 104769-104776. [CrossRef]

150. Jeon, D.S.; Park, J.H.; Kang, D.Y.; Dongale, T.D.; Kim, T.G. Forming-ready resistance random access memory using randomly pre-grown conducting filaments via pre-forming. Mater. Sci. Semicond. Process. 2020, 110, 104951-104956. [CrossRef]

151. Zhang, X.; Liu, S.; Zhao, X.; Wu, F.; Wu, Q.; Wang, W.; Cao, R.; Fang, Y.; Lv, H.; Long, S.; et al. Emulating Short-Term and Long-Term Plasticity of Bio-Synapse Based on Cu/a-Si/Pt Memristor. IEEE Electron. Device Lett. 2017, 38, 1208-1211. [CrossRef]

152. Lobov, S.A.; Mikhaylov, A.N.; Shamshin, M.; Makarov, V.A.; Kazantsev, V.B. Spatial Properties of STDP in a Self-Learning Spiking Neural Network Enable Controlling a Mobile Robot. Front. Neurosci. 2020, 14, 88-97. [CrossRef]

153. Kim, J.; Kim, C.-H.; Yun Woo, S.; Kang, W.-M.; Seo, Y.-T.; Lee, S.; Oh, S.; Bae, J.-H.; Park, B.-G.; Lee, J.-H. Initial synaptic weight distribution for fast learning speed and high recognition rate in STDP-based spiking neural network. Solid State Electron. 2020, 165, 107742-107747. [CrossRef]

154. Kim, K.Y.; Rios, L.C.; Le, H.; Perez, A.J.; Phan, S.; Bushong, E.A.; Deerinck, T.J.; Liu, Y.H.; Ellisman, M.A.; Lev-Ram, V.; et al. Synaptic Specializations of Melanopsin-Retinal Ganglion Cells in Multiple Brain Regions Revealed by Genetic Label for Light and Electron Microscopy. Cell Rep. 2019, 29, 628-644. [CrossRef] [PubMed]

155. Ilyas, N.; Li, D.; Li, C.; Jiang, X.; Jiang, Y.; Li, W. Analog Switching and Artificial Synaptic Behavior of $\mathrm{Ag} / \mathrm{SiO}_{\mathrm{x}}: \mathrm{Ag} / \mathrm{TiO}_{\mathrm{x}} / \mathrm{p}^{++}-\mathrm{Si}$ Memristor Device. Nanoscale Res. Lett 2020, 15, 30-40. [CrossRef] [PubMed] 
156. Pignatelli, M.; Tejeda, H.A.; Barker, D.J.; Bontempi, L.; Wu, J.; Lopez, A.; Palma Ribeiro, S.; Lucantonio, F.; Parise, E.M.; Torres-Berrio, A.; et al. Cooperative synaptic and intrinsic plasticity in a disynaptic limbic circuit drive stress-induced anhedonia and passive coping in mice. Mol. Psychiatry 2020, 2, 6868-6887. [CrossRef] [PubMed]

157. NagaJyothi, G.; Sridevi, S. High speed low area OBC DA based decimation filter for hearing aids application. Int. J. Speech Technol. 2019, 23, 111-121. [CrossRef]

158. Berdan, R.; Vasilaki, E.; Khiat, A.; Indiveri, G.; Serb, A.; Prodromakis, T. Emulating short-term synaptic dynamics with memristive devices. Sci. Rep. 2016, 6, 18639-18647.

159. Sun, L.; Hwang, G.; Choi, W.; Han, G.; Zhang, Y.; Jiang, J.; Zheng, S.; Watanabe, K.; Taniguchi, T.; Zhao, M.; et al. Ultralow switching voltage slope based on two-dimensional materials for integrated memory and neuromorphic applications. Nano Energy 2020, 69, 104472-104477. [CrossRef]

160. Chen, P.A.; Ge, R.J.; Lee, J.W.; Hsu, C.H.; Hsu, W.C.; Akinwande, D.; Chiang, M.H. An RRAM with a 2D Material Embedded Double Switching Layer for Neuromorphic Computing. In Proceedings of the 2018 IEEE 13th Nanotechnology Materials and Devices Conference (NMDC), Portland, OR, USA, 14-17 October 2018.

161. Shi, Y.; Pan, C.; Chen, V.; Raghavan, N.; Pey, K.L.; Puglisi, F.M.; Pop, E.; Wong, H.-P.; Lanza, M. Coexistence of volatile and non volatile resistive switching in $2 \mathrm{D} h$ - BN based electronic synapses. In Proceedings of the 2017 IEEE International Electron Devices Meeting (IEDM), San Francisco, CA, USA, 2-6 December 2017.

162. Sokolov, A.S.; Jeon, Y.-R.; Ku, B.; Choi, C. Ar ion plasma surface modification on the heterostructured $\mathrm{TaO}_{\mathrm{x}} / \mathrm{InGaZnO}$ thin films for flexible memristor synapse. J. Alloy. Compd. 2020, 822, 153625-153635. [CrossRef]

163. Wang, T.Y.; He, Z.Y.; Chen, L.; Zhu, H.; Sun, Q.Q.; Ding, S.J.; Zhou, P.; Zhang, D.W. An Organic Flexible Artificial Bio-Synapses with Long-Term Plasticity for Neuromorphic Computing. Micromachines 2018, 9, 239. [CrossRef]

164. Ohno, T.; Hasegawa, T.; Tsuruoka, T.; Terabe, K.; Gimzewski, J.K.; Aono, M. Short-term plasticity and long-term potentiation mimicked in single inorganic synapses. Nat. Mater. 2011, 10, 591-595. [CrossRef]

165. Ku, B.; Abbas, Y.; Kim, S.; Sokolov, A.S.; Jeon, Y.-R.; Choi, C. Improved resistive switching and synaptic characteristics using Ar plasma irradiation on the $\mathrm{Ti} / \mathrm{HfO}_{2}$ interface. J. Alloy. Compd. 2019, 797, 277-283. [CrossRef]

166. Wan, T.; Qu, B.; Du, H.; Lin, X.; Lin, Q.; Wang, D.W.; Cazorla, C.; Li, S.; Liu, S.; Chu, D. Digital to analog resistive switching transition induced by graphene buffer layer in strontium titanate based devices. J. Colloid Interface Sci. 2018, 512, 767-774. [CrossRef] [PubMed]

167. Baek, I.G.; Lee, M.S.; Seo, S.; Lee, M.J.; Seo, D.H.; Suh, D.S.; Park, J.C.; Park, S.O.; Kim, H.S.; Yoo, I.K.; et al. Moon, Highly scalable nonvolatile resistive memory using simple binary oxide driven by asymmetric unipolar voltage pulses, presented at the IEDM Technical Digest. In Proceedings of the IEEE International Electron Devices Meeting, San Francisco, CA, USA, 13-15 December 2004.

168. Zha, Y.; Wei, Z.; Li, J. Recent progress in RRAM technology: From compact models to applications. In Proceedings of the 2017 China Semiconductor Technology International Conference (CSTIC), Shanghai, China, 12-13 March 2017.

169. Karam, R.; Liu, R.; Chen, P.-Y.; Yu, S.; Bhunia, S. Security Primitive Design with Nanoscale Devices: A Case Study with Resistive RAM. In Proceedings of the 2016 International Great Lakes Symposium on VLSI (GLSVLSI), Boston, MA, USA, 18-20 May 2016.

170. Wang, W.; Li, Y.; Wang, M.; Wang, L.; Liu, Q.; Banerjee, W.; Li, L.; Liu, M. A hardware neural network for handwritten digits recognition using binary RRAM as synaptic weight element. In Proceedings of the the 2016 IEEE Silicon Nanoelectronics Workshop (SNW), Honolulu, HI, USA, 12-13 June 2016.

171. Yu, S.; Sun, X.; Peng, X.; Huang, S. Compute-in-Memory with Emerging NonvolatileMemories: Challenges and Prospects. In Proceedings of the 2020 IEEE Custom Integrated Circuits Conference (CICC), Boston, MA, USA, USA, 22-25 March 2020.

(C) 2020 by the authors. Licensee MDPI, Basel, Switzerland. This article is an open access article distributed under the terms and conditions of the Creative Commons Attribution (CC BY) license (http://creativecommons.org/licenses/by/4.0/). 